\title{
Motivation, resources and the organization of the school system*
}

\author{
Facundo Albornoz (University of Nottingham and CONICET) $†$ \\ Samuel Berlinski (Inter-American Development Bank and IZA) \\ Antonio Cabrales (University College London) $)^{\S}$
}

July 2016

\begin{abstract}
This paper studies a model where student effort and talent interact with parental and teachers' investments, as well as with school system resources. The model is rich, yet sufficiently stylized to provide novel implications. It can show, for example, that an improvement in parental outside options will reduce parental and school effort, which are partially compensated through school resources. In this way, by incorporating the behavioral responses of parents, teachers and policymakers, the paper provides a rationale for the existing ambiguous empirical evidence on the effect of school resources. The paper also provides a novel microfoundation for peer effects, with empirical implications for welfare and different education policies.
\end{abstract}

JEL-Classification: I20, I21, I28, J24

Key-words: education, incentives, school resources, parental involvement, school sorting, peer effects.

${ }^{*}$ We thank Andrés Erosa, Esther Hauk, Elena Iñarra, Gilat Levy, Diego Puga, Miguel Urquiola, and seminar participants at University of Birmingham, Columbia University, LACEA, IMDEA, XIII Urrutia Elejalde Summer School on Economics and Philosophy, Science Po, Seoul National University, Stockholm School of Economics and Warwick. Albornoz is grateful for support from the ESRC (RES-062-23-1360), Cabrales acknowledges the support the Spanish Ministry of Science and Technology under grant ECO201234581. The views expressed herein are those of the authors and should not be attributed to the Inter-American Development Bank, its Executive Directors, or the governments they represent.

${ }^{\dagger}$ facundo.albornoz@nottingham.ac.uk

${ }^{\ddagger}$ samuelb@iadb.org

§a.cabrales@ucl.ac.uk 


\section{Introduction}

Education policy is at the forefront of the social and political debate. The belief that education is a catalyst for a better and more equitable society ensures its role in the political agenda, in both developed and developing countries. As a consequence, a variety of policies and reforms are continuously being proposed with the objective of improving the outcomes of the education system. Surprisingly, the implementation and evaluation of these policies often overlook the changes in behavior they can induce in the actors involved in the education

process. For example, the debate about the role of school resources on student learning does not usually take into account behavioral responses from parents and school administrators. Similarly, school vouchers proposals generally disregard their influence on parental involvement in education. In this paper, we study a model of education where student learning effort and outcomes, parental and school behavior, and public resources devoted to education are endogenously determined in an integrated theoretical framework.

In our model, the determination of educational outcomes is a process involving four participants: children, parents, headmasters/teachers, and the policymaker. Each child chooses a certain level of effort devoted to learning. More able children obtain a higher learning outcome from every unit of effort. Altruistic parents and schools affect the effort decision through motivation schemes. However, inducing effort is costly for parents as well as for teachers. There is an opportunity cost for the time involved in setting up and executing the motivation plan, which may also include monitoring or helping children with their learning tasks (such as homework). Schools resources (class size, for example), which are determined by the policymaker, may affect the cost of motivating students in the same way as the talent of parents and teachers. This integrated framework provides an accurate description of the workings of the education process: parents, students and the education system interact in the determination of school resources,mas education quality, school and parent involvement and, through all these, on students outcomes. An advantage of our framework is its tractability, which allows us to analyze many important dimensions of the education process.

We start by analyzing a model where children are homogeneous in terms of innate ability and so are the parents' characteristics, such as their talent and opportunity cost of time. We find that the strength of parental and school involvement and the resources devoted to education increase with the student's innate ability. The results are less clear cut when we analyze the impact of rising opportunity cost of time associated with parental involvement in the learning process of their children. This introduces, in a natural way, the connection between labor market conditions and the direct involvement of parents in the education of 
their children. This link goes beyond the hourly wage. For example, it can also capture changes in opportunities and incentives for female participation in the labor market. In either case, the strength of parental involvement is decreasing in the opportunity cost of time.

The interaction between the school system and parental inputs is the reason why political considerations are important. As their opportunity cost of time increases, parents would like to rely more heavily on schools for motivating their children, which triggers actions by those responsible for the education system. The policymaker anticipates participant choices and satisfies parental wishes by increasing the resources devoted to education. Interestingly, the increase in school resources may not be accompanied by an overall improvement in educational attainment. Our model can predict "disappointingly" weak effects of school resources on student results even in situations where school resources do in fact affect learning cateris paribus. The weak effect can be rationalized because cateris paribus does not hold when resources increase. Parental involvement decreases because of a change in their opportunity costs. School resources increase to offset this reduction. ${ }^{1}$ These additional resources have in fact an effect, but this is not apparent because of concomitant changes in parental involvement in the education process.

We then allow for children to differ in terms of ability and parental opportunity costs of time, which leads to a number of insights. First, as the school determines their motivational policy for the average individual in the classroom, school involvement is positively affected by the mean ability of their students. In equilibrium, this affects the intensity of parental involvement. Thus, peer effects arise endogenously, as the choice of effort and motivational policy in equilibrium depend on the average ability of the student in the classroom. This effect is reinforced by the determination of school resources. The policy maker decides the level of resources optimally given the characteristics of the school attended by her/his political reference child; in our case, the median voter's child. Thus, the decision on school resources will be based on the average ability within this school and on the median child's ability. As a consequence, student effort depends on the mean abilities of peers at her/his school, plus the ability of the median child and of the peers of the median child. Our model generates in this way a microfoundation for peer effects, rather than assuming they come from some exogenous "contagion" process, as it is more common in the literature.

In this context, an increase in the opportunity cost of the median parent raises similar

\footnotetext{
${ }^{1}$ As an early example of the connection between parents' opportunity costs and school resources, Flyer and Rosen (1997) attribute the increase in school expenditures taking place in the United States during 1960-1990 to the growth in female labor-force participation.
} 
issues to those identified for the homogeneous case. However, the link between median child characteristics and individual effort generates a channel through which changes in the distribution of income (or talent) can affect the educational choices of households and schools. For example, an increase in the income of the median child's household will generate an increase of resources in the system. This will induce a positive effect on households in lower parts of the income distribution even if their incomes do not change. And the other way around, if the income of the median does not change (or it changes very little) in an environment where mean income is increasing markedly, there will be few changes in school outcomes (or even a regression) at a time when income appears to be fast increasing.

Our work is also related to the literature that studies the endogenous determination of class size. In Lazear (2001) class size is decided by schools according to student behavior. For example, when students have a shorter attention span (i.e., they can be distracted more easily) students should be sorted in smaller classrooms as they require closer attention. In Urquiola and Verhoogen (2009), schools differ in productivity and offer different quality levels (school size). As parents differ in earnings, sorting between schools with different class sizes arises naturally. Our model offers a complementary mechanism behind the determination of class size, which relies upon the interaction of parental and school motivation, which is partly determined by the government through the (strategic) choice of school resources.

We organize the paper as follows: In section 2, we set up the model. We characterize the equilibrium in section 3, where we discuss the interdependence between parental and school motivation systems, school resources and student performance. Section 4 discusses the connection of our results with the empirical literature. We conclude in section 5 .

\section{The model}

Our model has four participants: children, parents, headmasters, and the policymaker. ${ }^{2}$ There are different ways to specify the production function of human capital. We assume that learning requires innate talent and student effort. Parents and schools can affect this process through different interventions and actions. In the core of the paper, we emphasize parental and school interventions that affect student effort and therefore impact learning in

\footnotetext{
${ }^{2}$ With this structure we separate the actions of key participants in the education process, which allow us to theoretically identify the sources of multiple interacting sources of student effort. Teachers play a passive role and their preferences are subsumed under the headmaster actions, who, as explained in detail below, acts as an average teacher within each school. This simplification suppresses interesting potential principal-agent problems within schools. We do this to be able to focus better on the interactions between schools, families and the public sector.
} 
an indirect way.

Assuming that parents and schools can affect learning effort is consistent with what psychologists call "Achievement Goal Theory" (see, for example, Covington (2000)). According to this perspective, achievement goals influence the quality, timing and appropriateness of the students' engagement in their own learning (e.g., analyzing the demands of school tasks, planning and allocating resources to meet these demands, etc.). This effort together with innate ability affect the student's accomplishments. In our model, parents and teachers play a key role in influencing the student's achievement goals and, in turn, their effort.

Two kinds of goals have been predominantly studied in achievement goal theory: learning goals and performance goals. Learning goals refer to increasing one's competency, understanding and appreciation of what is learned. Performance goals involve outperforming others in tests or other achievement measures. In our model, parents and teachers can be thought as focusing on affecting learning goals.

Crucially, inducing effort is costly for parents as well as for schools. Both for parents and teachers, the main cost is the opportunity cost of the time involved encouraging students in the pursuit of learning goals. The cost for schools depends as well on the level of resources (for example, class size), which are determined by a policymaker. ${ }^{3}$

Student performance and children's short-term utility Human capital for child $i, H_{i}$, (measured in monetary terms) is a linear function of her effort, $e_{i}$, and a productivity parameter, $v_{i}$. In particular, we assume,

$$
H_{i}=v_{i} e_{i}
$$

In the core of the paper, we assume $v_{i}$ to be exogenous, which can therefore be interpreted as a measure of innate talent. We discuss the implications of different specifications of $H_{i}$ in Appendices A.1, A.2 and A.3.

Children internalize only imperfectly the effect of their effort on human capital. The role of parents and teachers is to induce students to exert costly effort through different motivational tools. In particular, we postulate that children's short-term utility is given by:

\footnotetext{
${ }^{3}$ We think there are good reasons to separate the actions of the policymaker and the teachers. To begin with, the model approximates more closely what we think is the actual decision process in reality. Perhaps more importantly, the current modeling choice helps to distinguish the channels through which the different parameters produce effects on, for example, teacher involvement and school resources. And since those variables are in principle observable and distinguishable, the separation of actions thus helps one of the objectives of the paper: to provide guidance for empirical research.
} 


$$
U_{S_{i}}=c_{1 i} e_{i}+c_{2 j} e_{i}-\frac{1}{2} e_{i}^{2}
$$

Exerting effort implies a cost that takes a quadratic form. Parent and teacher's involvement enters positively in the utility of the child, $c_{1 i} e_{i}+c_{2 j} e_{i}$, with the sub-index $j$ denoting the school attended by child $i . c_{1 i}\left(c_{2 j}\right)$ is a summary of the strength of parental (teacher) incentives for every unit of child's effort. Therefore, $c_{1 i} e_{i}+c_{2 j} e_{i}$ is the child's total reward for her effort. ${ }^{4,5}$

We are agnostic about the exact real life counterpart of $c_{1 i}\left(c_{2 j}\right)$. We think about it as a reduced form of a complex incentive structure that arises within families (schools). For example, $c_{1 i}$ include rewards such taking a child to the park, shopping or any leisure activity, but $c_{1 i}$ may also capture forms of motivation that require direct parental involvement in the learning process (e.g., helping with homework, going to museums, science fairs, or cultural activities) and that affect the value of acquiring knowledge. ${ }^{6}$ In other words, the role of parents (teachers) is to help children internalize the value of $e_{i}$ (and ultimately $H_{i}$ ) in their utility function.

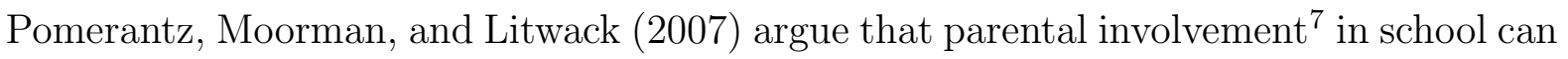
enhance children's school performance through either skill development (e.g., by instructing children) or motivational development (e.g., by providing intrinsic reasons for learning). Some motivational activities clearly play both roles. Take for example, helping with homework. On the one hand, it increases the productivity of the children effort (i.e., is tantamount to an increase in $v_{i}$ ). On the other hand, it increases the intrinsic value of studying for the

\footnotetext{
${ }^{4}$ This linear structure of rewards is analogous to the linear contracts that are widespread in reality. Bose, Pal, and Sappington (2011) justify the use of linear contracts by showing that, in a broad class of environments, the optimal linear contract always secures for the principal at least $90 \%$ of the expected profit secured under a fully optimal contract. Furthermore, Carroll (2013) considers an environment in which the principal is uncertain about what the agent can and cannot do and wants to write a contract that is robust to this uncertainty. He finds that, under very general circumstances, the optimal contract is linear.

${ }^{5}$ As we focus on effective motivation schemes, their costs both for parents and schools depend on student effort. This naturally introduces interactions between parents and schools. Framing these modeling choices in the distinction between intrinsic and extrinsic motivation, notice that assuming that the cost of $c_{1}$ is increasing in student effort is natural when considering $c_{1}$ as extrinsic motivation (e.g. helping with homework or rewarding activities). Our modeling choice for $c_{1}$ is potentially more restrictive in the case of intrinsic motivation. If increasing the intrinsic learning satisfaction of children making more effort requires spending more time with them (e.g. more hours reading books or carrying out cultural or scientific activities) then our assumption would also work well in the case of intrinsic motivation.

${ }^{6}$ Notice that we implicitly assume commitment problems away. If $c_{1}$ is interpreted as a reward, parents may in principle renege on their promises. To avoid this problem, it is possible to interpret the relationship between parents and children as a repeated game where reputation resolves the commitment issues.

${ }^{7}$ Following Hoover-Dempsey and Sandler (1997), parental involvement includes home-based activities related to children's learning (e.g., helping with homework, discussing school events) and school-based involvement (e.g., volunteering at school, attending school functions).
} 
child because parental involvement and concerns help children internalize the benefits of their effort. We focus on the motivational channel and leave for the appendix the discussion of parental interventions that directly affects the productivity of learning effort (Appendix A.1).

The additive specification for parent and teacher incentives implies that parents and school involvement are substitutes. ${ }^{8}$ Changing this assumption would yield no qualitative differences, as we discuss below and in Appendix A.3.

Of course, effort may also be obtained through negative reinforcement (punishment). In this case, we could have written the utility in the alternative way:

$$
U_{S_{i}}=-\left(c_{1 i}+c_{2 j}\right)\left(1-e_{i}\right)-\frac{1}{2} e_{i}^{2}=\left(c_{1 i}+c_{2 j}\right) e_{i}-\left(c_{1 i}+c_{2 j}\right)-\frac{1}{2} e_{i}^{2}
$$

As will be clear below, this utility induces the same optimal action $e_{i}$ from her as the one we examine. Thus, provided the costs of the two incentive systems can be written in the same way, there will be no difference in any equilibrium value. ${ }^{9}$

The parents' utility We assume that every parent has one child and that their utility is influenced by the sum of her performance and their own welfare, denoted by $W_{i}$ (also measured in monetary terms, as $\left.H_{i}\right) \cdot{ }^{10}$ Hence,

$$
U_{P_{i}}=H_{i}+W_{i}
$$

Parental welfare depends on the time spent at work or pursuing leisure activities. This is the total time $(T)$ available minus the time spent with the child as a consequence of the reward scheme $\left(t_{i}\right)$. The effective reward $\left(c_{1 i} e_{i}\right)$ depends on time $\left(t_{i}\right)$ and parental efficiency $\left(v_{P_{i}}\right)$ to generate a given effective reward, so that $c_{1 i} e_{i}=2 t_{i} v_{P_{i}}$. The parameter $v_{P_{i}}$ captures the fact that some parents are better at motivating than others and consequently they generate a larger reward for any given amount of time devoted to their children. That is, we associate productivity of parental involvement with some innate attribute, such as talent

\footnotetext{
${ }^{8}$ This formulation also assumes that effort is perfectly correlated with human capital. Nothing major would change in the model if the relationship between effort and output were noisy, provided effort were observable and contractible. We do not think this is an unrealistic assumption in the case of children. Nonobservability of effort with noisy output would be, of course, more complicated but we do not think any new insights would be gained by studying that case.

${ }^{9}$ In our setting, this is the case because we assume that parents care about $H$ but not about her short term utility.

${ }^{10}$ The utility function below does not internalize the child's cost of effort, so it is not purely "sympathetic". We have also done the computations with strictly sympathetic parents' utility and there are no significant changes.
} 
or persuasiveness. Implicitly, we are imposing that the time devoted inducing effort, for a given effective reward, is decreasing in the ability of the parent to generate it. In Appendix A.4, we study the effect of parental attention, where $v_{P_{i}}$ is derived endogenously and shown to depend on the opportunity cost of time.

Thus, letting $\psi_{i}$ be the opportunity cost of parent $i$ yields,

$$
W_{i}=\left(T-t_{i}\right) \psi_{i}=\left(T-\frac{c_{1 i} e_{i}}{2 v_{P_{i}}}\right) \psi_{i}
$$

and, therefore,

$$
U_{P_{i}}=v_{i} e_{i}+\left(T-\frac{c_{1 i} e_{i}}{2 v_{P_{i}}}\right) \psi_{i}
$$

Since $\psi_{i}$ is an opportunity cost of time for parents, it can be interpreted as a wage rate, although it can also be the value of leisure or something else. ${ }^{11}$ Hence, in the remainder of the paper we often refer to this parameter as parental income. ${ }^{12}$

REMARK 1 Parents also value (negatively) the (lump-sum) taxes that the government will need to levy in order to pay for school resources. ${ }^{13}$ We do not include them here explicitly in order to avoid an excess of notation. Given the quasi-linearity in income of utility and that taxation is already decided by the time parents choose their effort, the amount of those taxes do not affect the parental effort decision.

The school objective function According to Covington (2000), every classroom reflects rules that determine the basis on which students will be evaluated and how rewards will be distributed. We assume that teachers reward students based on individual learning expectations and not in a competition for one or a few prizes. The school rewards (and therefore the learning goals) are determined by the teachers' effort. ${ }^{14}$

\footnotetext{
${ }^{11}$ In our context, the marginal utility of money earned by parents is linear. Hence, the value of time for parents with high wages is larger. Things may be different with concave utility for money. In that case, low wage earners may have a higher opportunity cost of time. We are agnostic about which parents have the higher opportunity cost of time in reality.

${ }^{12}$ Notice we are not considering parental teaching time in this specification of parents' welfare. However, this would be easily incorporated into the analysis by assuming $H_{i}=T_{i}+W_{i}$, and $W_{i}=$ $\left(T-\frac{c_{1 i} e_{i}}{2 v_{P_{i}}}-\frac{1}{2} \frac{T_{i}^{2}}{v_{P_{i}}}\right) \psi_{i}$ where $t_{i}^{\prime}=T_{i}^{2} / 2 v_{P_{i}}$ represents the actual units of time a parent devotes to teaching and $T_{i}$ represents effective teaching time. Notice as well that this component is independent of student effort and has no interaction with $c_{1 i}$ and therefore it will have no effect on our results.

${ }^{13}$ Introducing proportional taxation would not be difficult. Proportional taxes would entail a reduction in the parental opportunity cost of effort $\psi_{i}$ and thus we can understand the impact of this kind of taxation by examining the effects of changes in $\psi_{i}$.

${ }^{14}$ For simplicity we assume that the costs of creating the rewards for the teachers are unrelated to student ability.
} 
We assume that the headmaster of a public school chooses the intensity of motivation (summarized in the parameter $c_{2 j}$ ) and therefore, teachers' effort, in order to maximize the sum of the average student performance and the welfare of the average teacher in the school. Of course, headmasters may be driven by other goals or use other statistics to measure school performance. In Appendices A.5 and A.6, we change the goal of maximizing average student performance in two crucial ways. In Appendix A.5, we study a case in which the headmaster cares more about a group of students according to their place in the distribution of human capital. ${ }^{15}$. We also study the case where headmasters are imposed an exogenous standards (Appendix A.6), such as, for example, that the average score be higher than a particular threshold (reference point). We show that the main messages of our analysis are robust to these variations in the headmaster's goals.

The average welfare of a teacher is determined by the difference between the total time available to him and the average time he devotes to his students, which we assume is a linear function of $c_{2 j} e_{i}$, and inversely related to $v_{T_{j}}$, the motivational ability of a representative teacher at school $j$. Thus, letting $\gamma$ be the opportunity cost of teachers' time, ${ }^{16}$

$$
U_{H M j}=\frac{1}{N_{j}} \sum_{i \in j} v_{i} e_{i}+\left(T-\frac{n_{j}}{N_{j}} \sum_{i \in j} \frac{c_{2 j} e_{i}}{2 v_{T_{j}}}\right) \gamma,
$$

where $N_{j}$ is the total number of students in school $j$ and $n_{j}$ is the number of students per classroom. Notice that $N_{j}$ in the denominator makes clear that students are randomly allocated to classes and $n_{j}$ in the numerator captures that larger classrooms involve more time for teachers. ${ }^{17}$

\footnotetext{
${ }^{15}$ More specifically, we study an alternative formulation where the headmaster cares about a weighted average of the mean and any order statistic of the distribution of human capital within the school. See Aucejo (2011) for an example of how teachers adjust their population targets within a classroom to changes in the goals imposed by the authorities.

${ }^{16} \mathrm{We}$ assume that the opportunity cost of the teacher $\gamma$ is unrelated to her talent $v_{T_{j}}$. This is done to simplify notation. Little is changed if $\gamma$ depends on $v_{T_{j}}$. It is worth pointing out that this is not the teacher's wage but rather the value of his alternative use of time which can either be leisure or the compensation he will get from, say, private tutoring. Notice as well that, as in the case of parents, we assume that the time devoted to motivate students depend linearly on their effort (e.g. highly motivated students require more challenging material or activities, which involve more time for their teachers).

${ }^{17}$ This specification implicitly assumes that teachers spend the same amount of time with students within a class. We think that this is reasonable, as a teacher that spent in a consistent way significantly larger amounts of time with some students than with others is likely to be challenged by parents, except for very especial children (say because they have an obvious disability). Even in countries with a history of discrimination, unequal treatment within a classroom occurs only under special circumstances (see e.g. Jain and Narayan (2011)).
} 
The policymaker objective function The policymaker maximizes the utility of the (median-voter) parent (denoted by $\bar{P}_{i}$ ) which, as discussed in remark 1, requires adding the cost of school resources $(1 / n)$. The decision about resources is taken and announced before parents and headmasters simultaneously decide their actions $\left(c_{1 i}\right.$ and $\left.c_{2 j}\right)$. Therefore, the cost of resources does not appear in $U_{P_{i}}$ or $U_{H M j}$ because parents and headmasters take them as given when making decisions about their involvement. These costs are paid by parents through general taxation, which parents care about, and are internalized by the policymaker when deciding $n$.

The cost depends on the number of classes to be manned. That is, the ratio of total number of students in the system, $N$, to the number of students per class, $n$. For simplicity, we assume that schools operate at full capacity and with the same class size so that $n_{j}=n$ for all $j$. Manning costs are assumed to be quadratic in the number of classrooms $N / n$. This can be justified by taking into consideration that the state has monopsony power in the market for teachers and faces a marginal cost function that increases in the number of teachers hired. This is so, for example, because to attract one more teacher the monopsonist has to pay an extra cost, since the marginal potential teacher needs a higher reward to be attracted to the profession.

Thus, we can represent the policymaker's preferences as,

$$
U_{P M}=U_{\bar{P}_{i}}-\frac{1}{N} \frac{\omega^{\prime}}{2}\left(\frac{N}{n}\right)^{2},
$$

where $\omega^{\prime}$ is a constant parameter summarizing the cost of the chosen class size and $\frac{1}{N} \frac{\omega^{\prime}}{2}\left(\frac{N}{n}\right)^{2}$ is the per capita cost of that class size. ${ }^{18}$ Our formulation assumes that schools are financed out of lump sum taxation and the government keeps a balanced budget. For ease of notation, in the remainder we denote $\omega=\omega^{\prime} N$, so that

$$
U_{P M}=U_{\bar{P}_{i}}-\frac{\omega}{2} \frac{1}{n^{2}},
$$

The structure of the game Summarizing, the policymaker announces first the policy variable $(n)$. After this announcement, parents and headmasters simultaneously decide their optimal levels of rewards per unit of effort $c_{1 i}$ and $c_{2 j}$, respectively. After observing parents' and schools' announcements, the children decide their optimal level of effort, $e_{i}$.

\footnotetext{
${ }^{18}$ Alternatively, the formulation can be reinterpreted by assuming that the average quality of teachers when hiring $k$ people is $1 / \sqrt{k}$. Hence, in order to man $N / n$ classrooms and keep the quality of teaching per classroom constant, $(N / n)^{2}$ teachers need to be hired. If $\omega^{\prime} / 2$ is the wage per employed person the total cost of $N / n$ classrooms is $\frac{\omega^{\prime}}{2}\left(\frac{N}{n}\right)^{2}$ and the cost per student $\frac{1}{N} \frac{\omega^{\prime}}{2}\left(\frac{N}{n}\right)^{2}$.
} 


\section{Equilibrium}

We solve the game by backward induction.

\subsection{Students', parents', and school choices}

From equation (2), it follows that the optimal student action is

$$
e_{i}=c_{1 i}+c_{2 j}
$$

Substituting this expression into the parents' utility, equation (3), we obtain

$$
U_{P_{i}}=\left(c_{1 i}+c_{2 j}\right) v_{i}+\left(T-\frac{1}{2 v_{P_{i}}} c_{1 i}\left(c_{1 i}+c_{2 j}\right)\right) \psi_{i} .
$$

The first-order conditions for the parents's problem is then

$$
v_{i}-\left(c_{1 i}+\frac{c_{2 j}}{2}\right) \frac{\psi_{i}}{v_{P_{i}}}=0 .
$$

Given that $U_{P_{i}}$ is concave in $c_{1 i}$, the optimal choice of the parent is

$$
c_{1 i}=\max \left\{\frac{v_{P_{i}} v_{i}}{\psi_{i}}-\frac{c_{2 j}}{2}, 0\right\},
$$

which by definition is non-negative since motivation is conducted in units of time.

It is clear from the expression for $c_{1 i}$ that the strength of parental involvement is increasing in the abilities of the child $\left(v_{i}\right)$ and the parent $\left(v_{P_{i}}\right)$, and decreasing in the parental opportunity cost of time $\left(\psi_{i}\right)$. Notice that $v_{P_{i}}$ and $\psi_{i}$ capture different aspects of the parental capability in motivating students. To simplify the parameter space, let us define the relative efficiency of parental motivation as

$$
\Upsilon_{P_{i}} \equiv \frac{v_{P_{i}}}{\psi_{i}}
$$

which is a sufficient statistic to describe relative efficiency of parents in extracting one unit of effort from their children. It follows that parental involvement increases in $\Upsilon_{P_{i}}$. Finally, equation (8) shows the negative relationship between $c_{1 i}$ and $c_{2 j}$. When motivation in the school is high, the gains from additional effort induced by parental motivation are smaller. We shall discuss below how both incentive schemes may compensate each other in responding to changes in $\psi_{i}, v_{P_{i}}$ and $v_{i}$.

At this point, the following clarification is in order: 
REMARK 2 The assumption of substitute rewards is not essential for the negative relationship between $c_{1 i}$ and $c_{2 j}$. A similar result is obtained with other specifications where parental and school efforts are complements. For example, when $H_{i}=v_{i} e_{i}{ }^{\alpha}$, with $\alpha<1$ and $U_{S_{i}}=c_{1 i} c_{2 j} e_{i}-\frac{1}{2} e_{i}^{2}$ rather than $U_{S_{i}}=c_{1 i} e_{i}+c_{2 j} e_{i}-\frac{1}{2} e_{i}^{2}$. (see Appendix A.3).

The driving force of our result is that the marginal cost of motivational efforts are increasing in student effort. If higher motivation at the school induces higher learning effort, then it is harder for parents to increase student effort of already school-motivated children. The same logic applies at the school level when parental motivation increases and induces greater student effort. Thus, the substitutability of motivation at school and at home emerges naturally from our modeling choice of motivation costs.

By substituting the optimal choice of children's effort into the utility function of the headmaster (4) we obtain:

$$
U_{H M j}=\frac{1}{N_{j}} \sum_{i \in j}\left(c_{1 i}+c_{2 j}\right) v_{i}+\left(T-\frac{n_{j}}{N_{j}} \sum_{i \in j} \frac{c_{2 j}\left(c_{1 i}+c_{2 j}\right)}{2 v_{T_{j}}}\right) \gamma
$$

It follows that an interior solution for the headmaster's optimization problem implies

$$
c_{2 j}=\frac{v_{T_{j}} \bar{v}_{j}}{\gamma n_{j}}-\frac{\bar{c}_{1 j}}{2}
$$

where $\bar{v}_{j}$ is the mean student ability and $\bar{c}_{1 j}$ is the mean parental reward for the students attending school $j$.

As in the case of the parents, we define the relative efficiency of teachers' motivation as

$$
\Upsilon_{T_{j}} \equiv \frac{v_{T_{j}}}{\gamma}
$$

We can interpret $\Upsilon_{T_{j}} \bar{v}_{j}$ as the school quality. Clearly, the talent of teachers plays a key role in defining schools' quality. Also, as in Epple and Romano (1998), the quality of schools depends on the average of the peers' talent. A higher average peer talent is associated with greater classroom motivation. This association, which will tend to amplify school differences in student performance, will be important in the emergence of peer effects. Also, raising opportunity costs for teachers and larger class sizes lead to lower levels of classroom motivation. It is, of course, through this latter channel that public resources affect student performance. Finally, a high level of parental involvement is associated with lower school incentives. 


\subsection{Equilibrium in the homogeneous case}

In order to solve for the first stage of the game, let us first assume that children, parents and teachers are all identical, so that $v_{i}=v, v_{P_{i}}=v_{P}$ and $\psi_{i}=\psi$ for all $i \in\{1, . ., N\}$ and $v_{T_{j}}=v_{T}$ for all $j$. In this case we have that $c_{1 i}=c_{1}$ for all $i$ and and $c_{2 j}=c_{2}$ for all $j$. Therefore, from (8), (9) and the definitions of $\Upsilon_{P_{i}}$ and $\Upsilon_{T_{j}}$, an interior solution for $c_{1}$ and $c_{2}$ implies

$$
c_{1}=\Upsilon_{P} v-\frac{c_{2}}{2}
$$

and

$$
c_{2}=\frac{\Upsilon_{T} v}{n}-\frac{c_{1}}{2}
$$

In the first stage, the policymaker considers the optimal level of school and parental involvement. After substituting (11) in (10) and plugging the resulting expression, together with (7), into (6) we obtain

$$
U_{P M}=\frac{2 v^{2}}{3}\left(\Upsilon_{P}+\frac{\Upsilon_{T}}{n}\right)+\left(T \psi-\frac{2 v^{2}}{9}\left(2 \Upsilon_{P}+\frac{\Upsilon_{T}}{n}-\frac{\Upsilon_{T}^{2}}{n^{2} \Upsilon_{P}}\right)\right)-\frac{\omega}{2} \frac{1}{n^{2}}
$$

As we show for the general heterogeneous case in the next section, preferences are unimodal in the policy parameter $n$ and we can apply the median-voter theorem to obtain the optimal level of $n$. The interior solution that results from maximizing the above expression with respect to $n$ is (assuming $\omega>4 v^{2} \Upsilon_{T}^{2} /\left(9 \Upsilon_{P}\right)$ )

$$
n=\frac{\omega-\frac{4 v^{2}}{9 \Upsilon_{P}} \Upsilon_{T}^{2}}{\frac{4 v^{2}}{9} \Upsilon_{T}}
$$

By inspection of (12) we immediately have:

Proposition 1 (comparative statics of class size) Class size is:

- increasing in the cost of manning classes $(\omega)$ and relative efficiency of parental motivation $\left(\Upsilon_{P}\right)$.

- decreasing in student ability $(v)$ and relative efficiency of teachers' motivation $\left(\Upsilon_{T}\right)$.

The effect of $\omega$ is economically obvious. The influence of teachers on schools resources is subtle. Remember that $\Upsilon_{T}$ increases in $v_{T}$ (teacher talent) and decreases in $\gamma$ (opportunity 
cost of teachers' time). The effect of $\gamma$ connects the commitment of teachers to government investment in school resources. If teachers find it harder to devote their time to school activities, the benefits of reducing the class size are marginally lower. On the other hand, teacher talent $\left(v_{T}\right)$ reinforces the benefits of investing in smaller classes. To our knowledge, these are empirically unexplored contrasting channels through which teacher characteristics influence public investment in school resources. Notice that the effect is even more complex if we allowed a positive correlation between teaching talent and the opportunity cost of teaching.

We also find that resources increase in student ability. This is a natural consequence of the fact that in our model, the marginal benefits for both parents and schools of strengthening teaching incentives increase with student talent.

Finally, the time parents can devote to their children's learning process has a direct (and observable) effect on school resources. Remember that $\Upsilon_{P}$ increases in $v_{P}$ (parental talent) and decreases in $\psi$ (opportunity cost of parents' time). For example, the post-war decades were characterized by a large increase in female labor-force participation. Arguably, this is compatible with an increase in $\psi$. This would result in our model in an decrease of $n$ (probably moderated by a concomitant increase in $\gamma$ ). Indeed, Flyer and Rosen (1997) find a connection between the rapid growth in public expenditures associated with elementary and secondary education in the US and the rising value of women's time. ${ }^{19}$

We substitute the optimal level of $n$ in equation (11) and then into equations (7) and (10) to obtain the equilibrium values of $c_{1}, c_{2}$ and $e$. These are:

$$
\begin{aligned}
& c_{1}=\frac{2 \Upsilon_{P} v}{3}\left(\frac{2 \omega-3\left(\frac{2 v \Upsilon_{T}}{3}\right)^{2} \frac{1}{\Upsilon_{P}}}{\omega-\left(\frac{2 v \Upsilon_{T}}{3}\right)^{2} \frac{1}{\Upsilon_{P}}}\right), \\
& c_{2}=\frac{2 \Upsilon_{P} v}{3}\left(\frac{3\left(\frac{2 v \Upsilon_{T}}{3}\right)^{2} \frac{1}{\Upsilon_{P}}-\omega}{\omega-\left(\frac{2 v \Upsilon_{T}}{3}\right)^{2} \frac{1}{\Upsilon_{P}}}\right),
\end{aligned}
$$

which imply:

\footnotetext{
${ }^{19}$ Between 1960 and 1990 the real costs of elementary and secondary education increased by 300 percent. The main cause of the increase in expenditures per student was rising school staff, with a halving of the number of the student-teacher ratios between 1950 and 1990. The paper studies further the connection between female labor force participation and student-teacher ratios using a panel of 50 US states during 4 decades. They found that increases in female labor force participation rates explain a significant part of both the level and growth in states' student-teacher ratios. Moreover, the findings are robust to other plausible explanations such as increases in unionization and changes in fertility patterns.
} 


$$
e=\frac{\frac{2 \Upsilon_{P} v}{3} \omega}{\omega-\left(\frac{2 v \Upsilon_{T}}{3}\right)^{2} \frac{1}{\Upsilon_{P}}} .
$$

From inspecting the above expressions, it becomes clear that obtaining positive values of $c_{1}, c_{2}$ imposes the following parameter restriction:

$$
3\left(\frac{2 v \Upsilon_{T}}{3}\right)^{2} \frac{1}{\Upsilon_{P}}>\omega>\frac{3}{2}\left(\frac{2 v \Upsilon_{T}}{3}\right)^{2} \frac{1}{\Upsilon_{P}} .
$$

Notice that the right-hand side of this condition also ensure positive values for $n$ and $e$. The following proposition gives a complete characterization of the main comparative statics:

\section{Proposition 2 (Comparative statics of motivation and student effort)}

1. The strength of parental motivation $\left(c_{1}\right)$ :

- increases in $v, \omega$ and in $\Upsilon_{P}$

- is decreasing with respect to $\Upsilon_{T}$

2. The strength of school motivation $\left(c_{2}\right)$ :

- increases in $\Upsilon_{T}$ and $v$

- is decreasing with respect to $\omega$ and $\Upsilon_{P}$

3. Student effort (e):

- increases in $\Upsilon_{T}$ and $v$.

- decreases in $\omega$.

- is ambiguous with respect to $\Upsilon_{P}$.

\section{Proof See Appendix C}

The resulting comparative statics with respect to $\Upsilon_{T}$ are simple to understand. An increase in teacher ability induces higher school involvement, which in turn induces higher student effort and reduces the strength of parental motivation. The effects of student innate ability, $v$, appears obvious from inspecting (14) and (15). However, this expression masks subtle behavioral feedbacks taking place between parents and schools. Using the 
parameter restrictions, we obtain that the sign of $\frac{\partial c_{1}}{\partial v}$ is negative. This means that talent reduces parental motivational efforts in equilibrium. But a lower $c_{1}$ induces higher motivation provision at the school $\left(c_{2}\right)$, which more than compensates lower levels of $c_{1}$.

The effect on effort $(e)$ of a decrease in $\Upsilon_{P}$ (resulting for example, from an increase in parental opportunity cost $\psi$ ) is ambiguous. First, a lower $\Upsilon_{P}$ translates into higher opportunity cost for parents to engage in motivational activities. Hence, $c_{1}$ decreases with the lower $\Upsilon_{P}$. The school system reacts to this by reducing $n$ and increasing $c_{2}$. The driving force for this result is that the policymaker devotes more resources to classroom education, which lowers the cost of inducing effort by the school. Conversely, lower class size and the consequent stronger school ethos, reduces the gain from inducing children's effort at home.

The optimal effort decision is equal to the sum of parental and school involvement. When the opportunity cost for parents increases, the resulting fall in parental involvement is not always fully compensated by the school system. Therefore, the net effect of a decrease in $\Upsilon_{P}$ on student performance may be negative. To see this:

$$
\frac{\partial e}{\partial \Upsilon_{P}}=\frac{\frac{2}{3} v \omega \Upsilon_{P}\left(9 \omega \Upsilon_{P}-8 v^{2} \Upsilon_{T}^{2}\right)}{\left(3 \omega \Upsilon_{P}-3\left(\frac{2 v \Upsilon_{T}}{3}\right)^{2}\right)^{2}}
$$

which implies that effort decreases with a lower $\Upsilon_{P}$ when

$$
\omega<\frac{8 v^{2} \Upsilon_{T}^{2}}{9 \Upsilon_{P}}=2\left(\frac{2 v \Upsilon_{T}}{3}\right)^{2} \frac{1}{\Upsilon_{P}} .
$$

Notice that from (16)

$$
3\left(\frac{2 v \Upsilon_{T}}{3}\right)^{2} \frac{1}{\Upsilon_{P}}>\omega>\frac{3}{2}\left(\frac{2 v \Upsilon_{T}}{3}\right)^{2} \frac{1}{\Upsilon_{P}}
$$

so that effort and school performance can be both increasing or decreasing within our parametric range.

\subsection{Equilibrium in the heterogeneous case}

We relax now the assumption of identical children. For expositional clarity, we define the following parameter:

$$
\bar{\Omega}_{j} \equiv \frac{1}{N_{j}} \sum_{i \in j} \Upsilon_{P_{i}} v_{i}
$$


In words, $\bar{\Omega}_{j}$ is the average at the school level of student ability times the ratio between parental motivational ability and their opportunity cost of time. Thus, each school $j$ is associated with a particular $\bar{\Omega}_{j}$.

To obtain the utility of the policymaker, we substitute (9) in (8) and plug the resulting expression into (7). This yields

$$
e_{i}^{*}=\Upsilon_{P_{i}} v_{i}+\frac{1}{3}\left(\frac{2}{n} \Upsilon_{T_{j}} \bar{v}_{j}-\bar{\Omega}_{j}\right)
$$

where $\bar{v}_{j}$ is the average student ability in school $j$. Thus, equation (6) becomes:

$$
\begin{aligned}
U_{P M}= & \left(\Upsilon_{P_{i_{M}}} v_{i_{M}}+\frac{1}{3}\left(\frac{2}{n} \Upsilon_{T_{j_{M}}} \bar{v}_{j_{M}}-\bar{\Omega}_{j_{M}}\right)\right) v_{i_{M}}+ \\
& +\left(T \psi_{i_{M}}-\frac{1}{2 \Upsilon_{P_{i_{M}}}}\left(\left(\Upsilon_{P_{i_{M}}} v_{i_{M}}\right)^{2}-\frac{1}{9}\left(\frac{2}{n} \Upsilon_{T_{j_{M}}} \bar{v}_{j_{M}}-\bar{\Omega}_{j_{M}}\right)^{2}\right)\right)-\frac{\omega}{2 n^{2}}
\end{aligned}
$$

where $M$ stands for the median voter and, therefore, $\bar{v}_{j_{M}}$ and $\bar{\Omega}_{j_{M}}$ are average characteristics in the school the median voter attends.

The following lemma states that preferences are unimodal in the policy parameter, $n$, and proves the validity of the median-voter theorem to determine school resources $(n)$ in our framework.

LEMma 1 Preferences of parents with respect to $n$ are unimodal for all parents if $\frac{1}{N_{j}} \sum_{i \in j} \Upsilon_{P_{i}} v_{i}<$ $3 \min _{i \in\{1, \ldots, N\}} \Upsilon_{P_{i}} v_{i}$.

\section{Proof Please see Appendix B.}

In words, the preferences of parents are unimodal if the minimum value of $\Upsilon_{P_{i}} v_{i}$ (the relative efficiency of parental motivation times the child's talent) is not lower than a third of the average value of $\Upsilon_{P_{i}} v_{i}$ in the population.

The first-order condition for the policymaker's maximization problem, provided an interior solution exists, is:

$$
\frac{\partial U_{P M}}{\partial n}=-\frac{2}{3} \frac{v_{i_{M}} \Upsilon_{T_{j_{M}}} \bar{v}_{j_{M}}}{n^{2}}-\frac{2}{9} \frac{\Upsilon_{T_{j_{M}}} \bar{v}_{j_{M}}}{\Upsilon_{P_{i_{M}}} n^{2}}\left(\frac{2}{n} \Upsilon_{T_{j_{M}}} \bar{v}_{j_{M}}-\bar{\Omega}_{j_{M}}\right)+\frac{\omega}{n^{3}}=0 .
$$

We can make the following assumption to reduce notational complexity: 


\section{Assumption 1}

$$
\Upsilon_{P_{i_{M}}} v_{i_{M}}=\bar{\Omega}_{j_{M}}
$$

This means that the product of parental and individual parameters for the median child is equal to the average ratio in her school. Using this assumption, equation (18) simplifies to:

$$
\frac{\partial U_{P M}}{\partial n}=-\frac{2}{3} \frac{v_{i_{M}} \Upsilon_{T_{j_{M}}} \bar{v}_{j_{M}}}{n^{2}}+\frac{2}{9} \frac{\Upsilon_{T_{j_{M}}} \bar{v}_{j_{M}} v_{i_{M}}}{n^{2}}-\left(\frac{2 \Upsilon_{T_{j_{M}}} \bar{v}_{j_{M}}}{3}\right)^{2} \frac{1}{\Upsilon_{P_{i_{M}}} n^{3}}+\frac{\omega}{n^{3}}=0
$$

and thus we obtain:

$$
n=\frac{\omega-\left(\frac{2 \Upsilon_{T_{j_{M}}} \bar{v}_{j_{M}}}{3}\right)^{2} \frac{1}{\Upsilon_{P_{i_{M}}}}}{\frac{4}{9} v_{i_{M}} \Upsilon_{T_{j_{M}}} \bar{v}_{j_{M}}}
$$

A positive class size requires $\omega-\left(\frac{2 \Upsilon_{T_{j_{M}}} \bar{v}_{j_{M}}}{3}\right)^{2} \frac{1}{\Upsilon_{P_{i_{M}}}}>0$.

Proposition 3 (Comparative statics of class size in heterogeneous schools) Class size is:

- increasing in the opportunity cost of manning classes ( $\omega)$ and the relative efficiency of the median voter's parent $\left(\Upsilon_{P_{i_{M}}}\right)$.

- decreasing in the ability of the median student $\left(v_{i_{M}}\right)$, the average talent of her/his peers $\left(\bar{v}_{j_{M}}\right)$; and the relative efficiency of teachers at the median voter's school $\left(\Upsilon_{T_{j_{M}}}\right)$.

Proof Immediate from equation 19.

Similarly to the homogeneous case (Proposition 1), class size increases with the cost of manning classes. Moreover, school resources are the result of political considerations and the characteristics of the median parents, his child and the school she attends are critical in the determination of class size as well. ${ }^{20}$ In particular, class size in public schools decreases when the opportunity cost of the median parent or his child's ability increases. Finally, class size is also decreasing in the quality of peers and teachers in the school the median child attends.

\footnotetext{
${ }^{20}$ This is because our modeling choice targets the median voter, as we show in Appendix A.5., this result could vary if the political process was different.
} 
From the derivation of (19) it is clear that parents of children with $v_{i}$ above $v_{i M}$ would like the level of school resources to be higher (e.g., smaller class sizes). So it would make sense for them to supply the school with extra resources, in the form of their own time and material resources. This has strong implications for segregation as it provides a reason for parents to look into private school provision. But even within public schools, they can choose to do so, if they are allowed. This could explain why parents choose to organize activities in schools, which can have a sizable effect on student achievement, as Anghel and Cabrales (2010) document for the case of Spain.

Using equation (19), the equilibrium values for $c_{1 i}, c_{2 j}$ and $e_{i}$ follow:

$$
\begin{gathered}
c_{1 i}=\Upsilon_{P_{i}} v_{i}-\frac{2}{3}\left(\frac{\Upsilon_{T_{j}} \bar{v}_{j}}{n}-\frac{\bar{\Omega}_{j}}{2}\right) \\
c_{2 j}=\frac{4}{3}\left(\frac{\Upsilon_{T_{j}} \bar{v}_{j}}{n}-\frac{\bar{\Omega}_{j}}{2}\right) \\
e_{i}=\Upsilon_{P_{i}} v_{i}+\frac{2}{3}\left(\frac{\Upsilon_{T_{j}} \bar{v}_{j}}{n}-\frac{\bar{\Omega}_{j}}{2}\right)
\end{gathered}
$$

The comparative statics with respect to individual characteristics are as follows:

\section{Proposition 4 (Comparative statics on individual characteristics)}

1. Parental motivation $\left(c_{1 i}\right)$ increases in $v_{i}$ and $\Upsilon_{P_{i}}$ and is decreasing with respect to $\Upsilon_{T_{j}}$

2. School motivation $\left(c_{2 j}\right)$ increases in $\Upsilon_{T_{j}}$.

3. Student effort $\left(e_{i}\right)$ increases in $v_{i}, \Upsilon_{T_{j}}$ and $\Upsilon_{P_{i}}$,

Proof Immediate from inspection of equations (20), (21) and (22)

These results are similar to those established for the homogeneous case (Proposition 2) with one exception, the contribution of parent ability $\Upsilon_{P_{i}}$. In Proposition 4 student effort $\left(e_{i}\right)$ increases in $\Upsilon_{P_{i}}$, whereas Proposition 2 stated that student effort $(e)$ was ambiguous with respect to $\Upsilon_{P}$. In both cases, parents have a direct impact on their children through their motivation efforts at home. The homogeneous case introduces an additional channel as

all the parents are the median parent. Thus, they trigger a counterbalancing effect through inducing more school resources. In the heterogeneous, this is only true for the median parent. 
The model provides a micro-foundation for the emergence of peer effects in the classroom without technological assumptions. ${ }^{21}$ The expression for (22) reveals that, in equilibrium, the performance of student $i$ is affected by the ability of her peers through different channels. First, $\bar{v}_{j}$ enters directly in Equation (22) increasing student effort. However, it also enters negatively through $\bar{\Omega}_{j}$. This captures the substitutability between parental and school motivation. To clarify the net effect of peers, let us denote the correlation coefficient between $\Upsilon_{P_{i}}$ and $v_{i}$ as $r_{P_{i} v_{i}}$. We also denote the standard deviation of $\Upsilon_{P_{i}}$ and $v_{i}$ as $s_{P_{i}}$ and $s_{v_{i}}$ respectively. Since,

$$
r_{P_{i} v_{i}}=\frac{\bar{\Omega}_{j}-\bar{v}_{j} \overline{\Upsilon_{P_{j}}}}{s_{P_{i}} s_{v_{i}}}
$$

we obtain:

$$
e_{i}=v_{i} \Upsilon_{P_{i}}+\frac{2}{3}\left(\frac{\bar{v}_{j} \Upsilon_{T_{j}}}{n}-\frac{r_{P_{i} v_{i}} s_{P_{i}} s_{v_{i}}+\bar{v}_{j} \overline{\Upsilon_{P_{j}}}}{2}\right)
$$

Now we can establish:

Proposition 5 (Peer effects) Assume that $r_{P_{i} v_{i}} \geq 0$, and that both the correlation $r_{P_{i} v_{i}}$ as well as standard deviations $s_{P_{i}}$ and $s_{v_{i}}$ are invariant to $\bar{v}_{j}$. Then, student effort increases in the ability of her own peers $\left(\bar{v}_{j}\right)$;

Proof Since $r_{P_{i} v_{i}}, s_{P_{i}}$ and $s_{v_{i}}$ are invariant to $\bar{v}_{j}$

$$
\frac{\partial e_{i}}{\partial \bar{v}_{j}}=\frac{2}{3}\left(\frac{\Upsilon_{T_{j}}}{n}-\frac{1}{2} \Upsilon_{P_{j}}\right)
$$

Note also that $c_{2 j} \geq 0$ requires that $\frac{\Upsilon_{T_{j}} \bar{v}_{j}}{n}-\frac{\bar{\Omega}_{j}}{2}=\frac{\Upsilon_{T_{j}} \bar{v}_{j}}{n}-\frac{r_{P_{i} v_{i}} s_{P_{i}} s_{v_{i}}+\bar{v}_{j} \overline{\Upsilon_{P_{j}}}}{2}=\frac{\Upsilon_{T_{j}} \bar{v}_{j}}{n}-\frac{1}{2} \bar{v}_{j} \overline{\Upsilon_{P_{j}}}-$ $\frac{r_{P_{i} v_{i}} s_{P_{i}} s_{v_{i}}}{2} \geq 0$. But, then, given that $r_{P_{i} v_{i}} \geq 0$

$$
\left(\frac{\Upsilon_{T_{j}}}{n}-\frac{1}{2} \overline{\Upsilon_{P_{j}}}\right) \bar{v}_{j} \geq \frac{\Upsilon_{T_{j}} \bar{v}_{j}}{n}-\frac{1}{2} \bar{v}_{j} \bar{\Upsilon}_{P_{j}}-\frac{r_{P_{i} v_{i}} s_{P_{i}} s_{v_{i}}}{2} \geq 0
$$

and the result follows.

Our model predicts that children who attend a school or class with better peers would improve their effort and educational outcomes. The following are the mechanisms operating

\footnotetext{
${ }^{21}$ We show in Appendix A.2 that introducing standard exogenous peer effects, where the human capital of the child is multiplied by a parameter capturing how performance is directly affected by the effort of peers, only amplifies the effects established in Proposition 5.
} 
in this situation. First, higher quality students increase school effort. However, parents do react to the increase in school efforts in the expected direction. Parents of children in schools with better (worse) peers reduce (increase) their involvement. Under the parametric assumptions of our model the parental reactions are not strong enough to offset the higher school effort. ${ }^{22}$ Thus, our model predicts a positive effect for a child that enters a higher quality school but this will be attenuated by parental behavioral responses.

Performance is also affected by the ability of the median student, the characteristics of her peers (ability and parental involvement) and her teacher's ability. We collect these effects on individual student effort coming from the political process in the following proposition:

Proposition 6 (Political effects on student effort) The student effort increases in the ability of the median student $\left(v_{i_{M}}\right)$; the ability of her/his teachers $\left(\Upsilon_{T_{j_{M}}}\right)$; her/his peers $\left(\bar{v}_{j_{M}}\right)$ and her/his parent $\left(\Upsilon_{P_{i_{M}}}\right)$.

Proof From equation 22 we know that $e_{i}$ is decreasing in $n$. The results then follows directly from proposition 3 .

As in the case of homogeneous parents and children, an economy-wide increase in parental opportunity costs induces a reduction of involvement by the parent (the effect of a decrease of $\Upsilon_{P_{i}}$ on $c_{1 j}$ is negative) which is partially compensated by the increased involvement of the school system (the effect of a decrease in $\Upsilon_{P_{i_{M}}}$ on $c_{2 j}$ is positive). But because of the link between the median child and individual effort, changes in the distribution of income (or talent) can affect outcomes as well. For example, a decrease in $\Upsilon_{P_{i_{M}}}$ will generate (for fixed $\theta_{j}$ ) an increase of resources (a decrease in $n$ ) which will have a positive effect on lower-income households even if their incomes do not change. Thus, a rising tide lifts all boats in this case. ${ }^{23}$ And the other way around, if $\Upsilon_{P_{i_{M}}}$ is unchanged (or almost, again for fixed $\theta_{j}$ ) in an environment where mean income is increasing markedly, there will be few changes in school outcomes (or even a regressive response, because of the negative reaction in $c_{1 i}$ of very high income households) at a time when GDP is increasing.

Equation (22) underlines an important issue regarding the generalization of partial equilibrium studies of stratification to the entire education system. In general, reduced form work (e.g., Duflo, Dupas, and Kremer (2011)) will focus on isolating the direct effect of $\bar{v}_{j}$

\footnotetext{
${ }^{22}$ This is straightforward to check by looking at the derivatives of $c_{1 i}$ and $c_{2 j}$ with respect to $\bar{v}_{j}$ in equations 20 and 21.

${ }^{23}$ Corcoran and Evans (2010) find that 12 to 22 percent of the increase in local school spending in the U.S. over the period 1970-2000 is attributable to rising inequality.
} 
on $H_{i}$. However, a generalized system for tracking students affects both $\bar{v}_{j}$ and $\bar{v}_{j_{M}}$. Thus, the general equilibrium implications may differ from the partial equilibrium ones through the political determination of the resources in the school system.

\section{Connections with the empirical literature}

Our results speak to two strands of the empirical literature that measure the causal effects of educational policy on student outcomes. First, to those who study the impact of changing school resources (e.g., reducing class-size). Second, to those who look at the effect of placing children in better schools. Here the word "better" is associated with some or all of the following characteristics: higher socio-economic status or ability peers, more skilled teachers, smaller class-sizes and better school facilities.

Isolating the effect of class size, or any other input, on educational outcomes in empirical work is hard because it essentially requires conditioning on all inputs. In general, what we can do is to find an exogenous source of variation in a market or shadow price - excluded by definition from the production function- which affects directly the input of interest and indirectly other inputs of the production function. Thus, we estimate what is called in the jargon a policy impact which contemplates the direct effect of the input of interest, say class-size, and changes in other inputs -behavioral responses.

Most of the literature focuses on the effect of educational policies on student outcomes as measured by contemporaneous and subsequent test-scores, school placement and high-school graduation and health, employment and income in adult life. Very few studies, however, measure thoroughly the behavioral responses from teachers and parents. ${ }^{24}$ Precisely, one main contribution of this paper is to establish that the policy effect is the result of a complex process of interaction among multiple actors. This suggests the need for empirical studies to unpack the policy impact by disentangling the different channels uncovered by our model.

In our case, an increase in the opportunity cost of parents or a fall in the cost of manning classes result in lower class-size. A fall in the cost of manning classes leads to an unambiguous increase in effort and an improvement in student performance. Most credible studies of the effect of class-size rely on reductions in the cost of manning classes either because of rules governing maximum class-size (Angrist and Lavy, 1999; Fredriksson, Öckert, and Oosterbeek, 2013, e.g.) which allocate resources to fund additional classes or because researchers facilitate

\footnotetext{
${ }^{24}$ This is somewhat surprising as there is a an extensive literature in public finance on the flypaper effect; see Hines and Thaler (1995, e.g.) for a review and Das, Dercon, Habyarimana, Krishnan, Muralidharan, and Sundararaman (2013) and Jacoby (2002) for examples related to schooling.
} 
the funds to operate smaller class-sizes (Krueger, 1999, e.g.). ${ }^{25}$ These studies find that lower class-sizes lead to better student outcomes.

Surprisingly, however, there are few credible estimates of the behavioral responses of teachers and parents to smaller class-sizes. An exception is the work of Fredriksson, Öckert, and Oosterbeek (2013, Forthcoming) that measure the causal effect of class-size on individual outcomes in childhood and adulthood in Sweden. They find that smaller class-sizes lead to better outcomes in the short, medium and long run (Fredriksson, Öckert, and Oosterbeek, 2013). Consistent with the predictions of our model, Fredriksson, Öckert, and Oosterbeek (Forthcoming) find that larger class-sizes cause parents to spend more time helping children with homework but teachers tend to places a larger onus on their students under these circumstances. This new evidence suggests that behavioral responses should be taken into account in research design and ultimately in the evaluation of public policy.

Looking now at the impact of allocating children to better schools, in our model, an improvement in the average ability of school peers leads to higher school effort. However, parents of children in schools with better (worse) peers reduce (increase) their involvement. Under the parametric assumptions of our model the parental reactions are not strong enough to offset the higher school effort. Thus, our model will predict a positive effect for a child that enters a higher quality school but this will be attenuated by parental behavioral responses.

There is a large number of studies about the effect of going to a better school. For identification purposes all of these studies involve a layer of randomness in the allocation of children to schools ${ }^{26}$ which is exploited for identification purposes. Some papers analyze national or municipal policies where all the students in the system are allocated using a given rule while others looked at participation in charter schools, targeted voucher programs or admissions to highly selective institutions. Cullen, Jacob, and Levitt (2006) and Pop-Eleches and Urquiola (2013) find that the parents of children that enter elite tracks or schools with better peers spend less time helping their children with homework than the counterfactual. This is consistent with our model.

On the final outcome there are indeed some studies which find positive effects of better peers on student outcomes (Pop-Eleches and Urquiola, 2013; Deming, Hastings, Kane, and

\footnotetext{
${ }^{25}$ Jackson et al (2016) present credible causal evidence of the effect of increasing school resources in general by using school finance reforms in the US. They find that the reforms were associated with reductions in student-to-teacher ratios, increases in teacher salaries and longer school years which eventually lead to sizable improvements in years of completed education and adult wages.

${ }^{26}$ In some cases schools are oversubscribed so there is a lottery for the allocation of students to schools. In others assignment to schools depends on an entry score and identification relies in comparing those students marginally in and out of the school.
} 
Staiger, 2014; Dobbie and Jr., 2011, e.g.). There are, however, others which find no effects (Abdulkadiroglu, Angrist, and Pathak, 2014; Cullen, Jacob, and Levitt, 2006; Clark, 2010, e.g.). ${ }^{2728}$ Within the confines of our model this discrepancy may be due to parametric differences, which lead to much stronger countervailing effects in the environments which find no effects. Thus, our general message is that in order to interpret the reduced form effect on outcomes of better schools one needs to consider the behavioral responses of the different actors. This would help to understand the contexts in which the results found in a particular paper carry to other contexts.

Finally, our model suggests a connection between parental opportunity cost of time, school peers, teacher characteristics and the determination of school resources. A related point appears in Besley and Case (2000), education policies are not exogenous to the political process. The literature, however, is yet to provide credible empirical estimates on how these parameters may affect the choices politicians make about the allocation of school resources.

\section{Concluding remarks}

In this paper, we study a model of education where student learning effort and outcomes, parental and school behavior, and public resources devoted to education are endogenously determined in an integrated and tractable framework. Our model, provides a rationale for why the evaluation of educational interventions often provides mixed results. Beyond rationalizing this phenomenon ex-post, the paper serves as a warning: many evaluation exercises in education may be seriously compromised by issues of external validity due to a lack of knowledge of behavioral aspects provoking and responding to changes in education policy. Indeed, we show that the effects of changing educational inputs on educational outcomes depend crucially on the sources of variation that cause this change. For this reason, it may be hard to formulate education policy based on a menu of evaluation results.

The model also provides a microfoundation for peer effects. Groups of children with higher average ability are more "profitable" to manage by teachers, who as a consequence exert more effort in them. Then, any child will benefit from their presence in the school.

\footnotetext{
${ }^{27}$ It is worth pointing out, however, that in none of the studies we describe it is possible to isolate whether the effect of going to a better school is driven by, for example, better peers, better teachers, better facilities, etc. To the best of our knowledge, only Duflo, Dupas, and Kremer (2011) provide an environment in their regression discontinuity design where everything else is kept constant. They find that there is no difference between the lowest scoring students assigned to the high achievement class and the highest scoring students assigned to the low-achievement class.

${ }^{28}$ Epple and Romano (2011) is an excellent survey of theoretical models, econometric estimation problems and empirical results on peer effects in education.
} 
Peer effects, as in other models, may produce an incentive for sorting. It is not hard to show that in some circumstances (e.g., when teaching technology favors low variance classrooms) sorting could be Pareto improving. However, if the benefits from sorting are not evenly distributed its welfare effects are potentially ambiguous since new classroom composition can trigger lower motivation incentives from parents and at the school. We explore the effects of sorting in Albornoz, Berlinski, and Cabrales (2010), where governments can implement policies leading to sorting according to talent, cultural preferences or religious beliefs. We show that the resulting changes in classroom composition may lower parents' and school motivation efforts and reduce resources in disadvantaged classes. As a consequence, the existing evaluations of these policies may need to be re-evaluated upon this light.

The richness of our environment can be used to look at other important questions. For example, it could be used to explore the consequences of increasing school choice through vouchers (see e.g. Epple and Romano (1998), Urquiola and Verhoogen (2009)) in a mixed education system with private schools. The implementation of a voucher scheme subsidizing private schools would involve changes in classroom composition which can trigger behavioral response attenuating or reinforcing its effects. In particular, vouchers could induce peers with higher talent to leave the public school. ${ }^{29}$ This could imply a decline in incentives and resources received by the students who stay in the public school. ${ }^{30}$ This result would follow mechanically from assuming the existence of peer effects as in most of the literature (e.g., Benabou (1993)). In our model, where peer-effects are endogenously generated by the interaction of parents and the school system, there would be an amplification effect via the reaction of school resources and incentives to changes in the average ability of the peers. We explore further the policy implications of our model in an ongoing project.

Furthermore, we have studied a static game. A dynamic version of the model is likely to have consequences on two dimensions. First, a time varying comparative advantage of parents and schools in motivating children may imply that the optimal path of parental, school and government investment may differ over time. Second, a dynamic model will allow for learning about the child's type which might lead to important consideration about the optimal timing of investment akin to those uncovered in the literature of R\&D learning (see, e.g. Grenadier and Weiss (1997)).

\footnotetext{
${ }^{29}$ This effect has been empirically uncovered by many studies. See for example, Howell and Peterson (2002) for the case of the US, Hsieh and Urquiola (2006), for Chile or Ladd (2002) for New Zealand.

${ }^{30}$ Altonji, Huang, and Taber $(2004,2010)$ provides evidence of this effect for the case of the U.S. MacLeod and Urquiola (2015) study a model where individuals sort between schools for reputational reasons, because employers observe average school performance. In that model, interestingly, the concern to have good peers induces too much test preparation before admission, but too little study effort after admission.
} 
Finally, the political aspects of school choice are barely scratched in this paper. Since the political authorities have a single instrument, school resources, and preferences over this instrument are single-peaked, we can resort to the median voter theorem in discussing the policymaker's choice. If there were more instruments (say, because the level of funding of charter schools is an electoral issue) more challenging (and more interesting) political interactions involving education could be studied (as in, for example, Boldrin and Montes (2005) or Levy (2005)). 


\section{References}

Abdulkadiroglu, A., J. Angrist, and P. Pathak (2014): "The Elite Illusion: Achievement Effects at Boston and New York Exam Schools," Econometrica, 82(1), 137-196.

Albornoz, F., S. Berlinski, and A. Cabrales (2010): "Incentives, resources and the organization of the school system," Discussion paper, CEPR Discussion Papers.

Altonji, J., C. Huang, and C. Taber (2010): "Estimating the Cream Skimming Effect of School Choice," NBER Working Paper No. 16579.

Altonji, J., C.-I. Huang, and C. Taber (2004): "Estimating the Cream Skimming Effect of Private School Vouchers on Public School Students," Yale University.

Anghel, B., and A. Cabrales (2010): "The Determinants of Success In Primary Education in Spain," FEDEA.

Angrist, J., And V. Lavy (1999): "Using Maimonides' Rule to Estimate the Effect of Class Size on Scholastic Achievement," The Quarterly Journal of Economics, 114, 533-575.

Aucejo, E. M. (2011): "Assessing the Role of Teacher and Student Interactions," Discussion paper, Working paper.

Benabou, R. (1993): "Workings of a City: Location, Education, and Production," The Quarterly Journal of Economics, 118(3), 619-652.

Besley, T., And A. Case (2000): "Unnatural experiments? Estimating the incidence of endogenous policies," Economic Journal, 110(467), F672-F694.

Boldrin, M., And A. Montes (2005): "The Intergenerational State: Public Education and Pensions," The Review of Economic Studies, 72(3), 651-664.

Bose, A., D. Pal, and D. Sappington (2011): "On the Performance of Linear Contracts," The Journal of Economics and Management, 20(1), 159-197.

Carroll, G. (2013): "Robustness and linear contracts," American Economic Review.

Clark, D. (2010): "Selective Schools and Academic Achievement," The B.E. Journal of Economic Analysis \&3 Policy, 10(1 (Advances)), 9.

Corcoran, S., and W. N. Evans (2010): "Income inequality, the median voter, and the support for public education," Discussion paper, National Bureau of Economic Research. 
Covington, M. (2000): "Goal Theory, Motivation, and School Achievement: An Integrative Review," Annual Review of Psychology, pp. 171-200.

Cullen, J., B. JaCOB, And S. LevitT (2006): "The effect of school choice on participants: evidence from randomized lotteries," Econometrica, 74(5), 1191-1230.

Das, J., S. Dercon, J. Habyarimana, P. Krishnan, K. Muralidharan, and V. Sundararaman (2013): "School Inputs, Household Substitution, and Test Scores," American Economic Journal: Applied Economics, 5(2), 29-57.

Deming, D., J. Hastings, T. J. Kane, and D. O. Staiger (2014): "School Choice, School Quality, and Postsecondary Attainment," American Economic Review, 104(3), 9911013.

Dobbie, W., And R. F. JR. (2011): "Are High-Quality Schools Enough to Increase Achievement Among the Poor? Evidence from the Harlem Children's Zone," American Economic Journal: Applied Economics, 3(3), 158-187.

Duflo, E., P. Dupas, and M. Kremer (2011): "Peer Effects, Teacher Incentives, and the Impact of Tracking: Evidence from a Randomized Evaluation in Kenya," American Economic Review, 101(5), 1739-74.

Epple, D., and R. Romano (1998): "Competition between Private and Public Schools, Vouchers, and Peer-Group Effects," The American Economic Review, 88(1), 33-62.

Epple, D., And R. Romano (2011): "Peer effects in education: A survey of the theory and evidence," Handbook of social economics, 1(11), 1053-1163.

Flyer, F., And S. Rosen (1997): "The New Economics of Teachers and Education," Journal of Labor Economics, 15(1), 104-39.

Fredriksson, P., B. ÖCKert, and H. Oosterbeek (2013): "Long-Term Effects of Class Size," Quarterly Journal of Economics, 128(1), 249-285.

(Forthcoming): "Parental responses to public investments in children: Evidence from a maximum class size rule," Journal of Human Resources.

Grenadier, S. R., ANd A. M. Weiss (1997): "Investment in technological innovations: An option pricing approach," Journal of financial Economics, 44(3), 397-416. 
Hines, J. R., And R. H. Thaler (1995): "Anomalies: The flypaper effect," The Journal of Economic Perspectives, 9(4), 217-226.

Hoover-Dempsey, K., And H. Sandler (1997): "Why Do Parents Become Involved in Their Children's Education?," Review of Educational Research, pp. 3-42.

Howell, W., And P. Peterson (2002): The Education Gap. Brookings Press, Washington, DC.

Hsieh, C.-T., And M. Urquiola (2006): "The effects of generalized school choice on achievement and stratification: Evidence from Chile's voucher program," Journal of Public Economics, 90(8-9), 1477-1503.

JACOBY, H. G. (2002): "Is there an intrahousehold flypaper effect? Evidence from a school feeding programme," The Economic Journal, 112(476), 196-221.

Jain, T., And T. NARAYAN (2011): "Incentive to discriminate? An experimental investigation of teacher incentives in India," An Experimental Investigation of Teacher Incentives in India (April 20, 2011).

Krueger, A. (1999): "Experimental Estimates of Education Production Functions," Quarterly Journal of Economics, 114, 497-532.

LADD, H. (2002): "School Vouchers: A Critical View," Journal of Economics Perspectives, $16(4), 3-24$.

LAzEAR, E. P. (2001): "Educational Production," The Quarterly Journal of Economics, 116(3), 777-803.

Levy, G. (2005): "The Politics of Public Provision of Education," The Quarterly Journal of Economics, 120(4), 1507-1534.

MacLeod, W. B., and M. Urquiola (2015): "Reputation and school competition," The American Economic Review, 105(11), 3471-3488.

Pomerantz, E., E. Moorman, and S. Litwack (2007): "The How, Whom, and Why of Parents' Involvement in Children's Academic Lives: More Is Not Always Better," Review of Educational Research, 77(3), 373-410.

Pop-Eleches, C., And M. Urquiola (2013): "Going to a better school: Effects and Behavioral Responses," American Economic Review, 130(4), 1289-1324, Frontiers. 
Urquiola, M., and E. Verhoogen (2009): "Class-Size Caps, Sorting, and the Regression-Discontinuity Design," American Economic Review, 99(1), 179-215.

\section{A Appendix A: Extensions and robustness checks}

A crucial insight of our paper is that we should consider the behavioral responses of parents when evaluating changes in educational policy. In this appendix, we discuss how some of the modeling assumptions we made may affect the direction of these responses.

\section{A.1 Monetary investments in learning}

If school performance is determined alone by effort and talent, then it is natural to consider, as we did so far, that parents induce more effort through time-consuming motivational and monitoring activities. However, student outcomes may also be enhanced by other activities with a direct effect on learning, such as paying for private tuition. Dealing with this concern requires changes to the production function of human capital $\left(H_{i}\right)$. Therefore, we study now the case where learning outcomes are a function of monetary resources paid by parents.

To study how parental (monetary) education investments interplay with school (monetary) resources, schools are also allowed to allocate monetary resources to enhance learning. School (monetary) resources can be used, for example, to hire personal tutors or teaching assistants. For simplicity, we assume that school monetary resources are chosen by the policy maker. We also assume that monetary education resources are substitute to time effort in order to focus on their direct effect. Also, this assumption greatly simplifies the analysis since we can abstract from the determination of student effort.

In particular, let $H_{i}, U_{P i}$ and $U_{P M}$ be specified in the following way: ${ }^{31}$

$$
\begin{gathered}
H_{i}=\left(g_{i}+g_{P M}\right)^{1 / 2} \\
U_{P_{i}}=2\left(g_{i}+g_{P M}\right)^{1 / 2}-\frac{\eta_{i}}{2} g_{i}^{2} \\
U_{P M}=2 \sum_{i=1}^{n}\left(g_{i}+g_{P M}\right)^{1 / 2}-\frac{\varphi}{2} g_{P M}^{2}
\end{gathered}
$$

\footnotetext{
${ }^{31}$ For simplicity in this discussion we subsume school decisions with those of the policymaker.
} 
Where $g_{i}$ and $g_{P M}$ represent monetary investment in learning by parents and the school, respectively. The first order conditions of this problem are:

$$
\begin{aligned}
\left(g_{i}+g_{P M}\right)^{-1 / 2} & =\eta_{i} g_{i} \\
\sum_{i=1}^{n}\left(g_{i}+g_{P M}\right)^{-1 / 2} & =\varphi g_{P M}
\end{aligned}
$$

It readily follows that the policy maker increases $g_{P M}$ as a response of parents lowering $g_{i}$, and vice versa. If we take the analysis further, we obtain that in equilibrium:

$$
\begin{aligned}
g & =\left(\frac{1}{\eta}\right)^{\frac{2}{3}}\left(\frac{1}{\frac{n \eta}{\varphi}+1}\right)^{\frac{1}{3}} \\
g_{P M} & =\frac{n}{n \varphi^{\frac{2}{3}}+\frac{\varphi}{\eta^{\frac{1}{3}}}}
\end{aligned}
$$

An important implication behind these expressions is that the impact evaluation of public education expenditure requires taking into account parental investments, even if we consider that parental monetary involvement as a substitute for learning effort; we obtain a similar insight when we looked at the effect of motivation on student learning effort.

\section{A.2 Direct peer effects}

Peer effects in our model arise so far only because a class with better (worse) students induces the teacher to provide higher (lower) effort. But in reality it is plausible that students benefit (or harm) one another directly, and not only through their effect on the effort level of teachers, or the investment of policymakers. We now also add this kind of direct peer effects, where the human capital of a child is affected by the average effort in the school. Specifically, let us assume that

$$
H_{i}=v_{i} e_{i}\left(1+\lambda \bar{e}_{1 j}\right)
$$

Following an analysis similar than at the core of the paper we obtain

$$
\begin{aligned}
c_{1 i} & =\max \left\{\Upsilon_{P_{i}} v_{i}\left(1+\lambda \bar{e}_{1 j}\right)-\frac{c_{2 j}}{2}, 0\right\} \\
c_{2 j} & =\frac{\Upsilon_{T_{j}} \bar{v}_{j}}{n_{j}}\left(1+\lambda \bar{e}_{1 j}\right)-\frac{\bar{c}_{1 j}}{2}
\end{aligned}
$$


and hence

$$
\begin{aligned}
\bar{c}_{1 j} & =\overline{\Upsilon_{P_{i}} v_{i}}\left(1+\lambda \bar{e}_{1 j}\right)-\frac{c_{2 j}}{2} \\
\bar{e}_{1 j} & =\frac{1}{\frac{3}{2}-\overline{\Upsilon_{P_{i}} v_{i}} \lambda-\frac{\Upsilon_{T_{j} \bar{v}_{j}}}{n_{j}} \lambda}\left(\overline{\Upsilon_{P_{i}} v_{i}}+\frac{\Upsilon_{T_{j}} \bar{v}_{j}}{n_{j}}\right) \\
e_{i}^{*} & =\Upsilon_{P_{i}} v_{i}+\frac{\Upsilon_{T_{j}} \bar{v}_{j}}{n_{j}}+\frac{\lambda \Upsilon_{P_{i}} v_{i}+\lambda \frac{\Upsilon_{T_{j}} \bar{v}_{j}}{n_{j}}-\frac{1}{2}}{\frac{3}{2}-\overline{\Upsilon_{P_{i}} v_{i}} \lambda-\frac{\Upsilon_{T_{j} \bar{v}_{j}}}{n_{j}} \lambda}\left(\overline{\Upsilon_{P_{i}} v_{i}}+\frac{\Upsilon_{T_{j}} \bar{v}_{j}}{n_{j}}\right)
\end{aligned}
$$

As can be readily seen by inspecting the previous expressions, the direct peer effects make the impact of all the exogenous variables larger, but there are no additional qualitative effects.

\section{A.3 Parent and teacher complements}

Let's assume that parents' and teachers' efforts are complements so that

$$
c=c_{1} c_{2}
$$

Further, for tractability reasons, human capital is characterized now by $H_{i}=v_{i} e_{i}^{\alpha}$, with $\alpha<1$.

The optimal student action is now,

$$
e=c_{1} c_{2}
$$

Substituting this expression into the parents' utility we obtain

$$
U_{P}=\left(c_{1} c_{2}\right)^{\alpha} v+\left(T \psi-\frac{c_{1}\left(c_{1} c_{2}\right)}{2 \Upsilon_{P}}\right) \text {. }
$$

The first-order condition for the parents's problem is then

$$
\begin{gathered}
\alpha c_{1}^{\alpha-1} c_{2}^{\alpha} v-c_{1} c_{2} \frac{1}{\Upsilon_{P}}=0 . \\
\alpha c_{2}^{\alpha} v-c_{1}^{2-\alpha} c_{2} \frac{1}{\Upsilon_{P}}=0
\end{gathered}
$$


Given that this condition is sufficient, the optimal choice of the parent is

$$
c_{1}=\left(\frac{\alpha \Upsilon_{P} v}{c_{2}^{1-\alpha}}\right)^{\frac{1}{2-\alpha}}
$$

which is always non-negative given that producing the rewards requires investing parental time. By substituting the optimal choice of children's effort into the utility function of the headmaster we obtain:

$$
U_{H M}=\left(c_{1} c_{2}\right)^{\alpha} v+\left(T \gamma-\frac{n c_{2}\left(c_{1} c_{2}\right)}{2 \Upsilon_{T}}\right)
$$

It follows that an interior solution for the headmaster's optimization problem implies

$$
c_{2}=\left(\frac{\alpha \Upsilon_{T} v}{n c_{1}^{1-\alpha}}\right)^{\frac{1}{2-\alpha}}
$$

From (24) and (25) it is clear that the equilibrium values of $c_{1}$ and $c_{2}$ move in opposite directions even though in this version of the model they are technological complements.

\section{A.4 Endogenous quality of parental involvement}

In the core of the paper, we associated productivity of parental motivation with something innate (exogenous), like talent or capacity of persuasion. However, for a given unit of time investment (and a given talent), parental motivation may also differ according to different levels of attention or commitment to the task of motivating student effort. In our model, this dimension can be captured by treating $\Upsilon_{P_{i}}$ as an endogenous variable. Consider that the utility of parents is now defined by:

$$
U_{P_{i}}=v_{i} e_{i}+\left(T \psi_{i}-\frac{c_{1 i} e_{i}}{2 \Upsilon_{P_{i}}}\right)-\frac{1}{2} \Upsilon_{P_{i}}^{2},
$$

where $\Upsilon_{P_{i}}$ subsumes the quality of parental effort (attention) and involves quadratic costs. For concreteness, we abstract from motivation provided by the school system. ${ }^{32}$ This implies that effort is solely determined by $c_{1 i}$ and thus

$$
U_{P_{i}}=v_{i} c_{1 i}+\left(T \psi_{i}-\frac{c_{1 i}^{2}}{2 \Upsilon_{P_{i}}}\right)-\frac{1}{2} \Upsilon_{P_{i}}^{2}
$$

\footnotetext{
${ }^{32}$ A similar analysis would carry over with similar insights if we dealt with endogenous quality of effort for teachers.
} 
From the first order condition with $c_{1 i}$ and $\Upsilon_{P_{i}}$ we obtain:

$$
\begin{aligned}
v_{i}-\frac{c_{1 i}}{\Upsilon_{P_{i}}} & =0 \\
\frac{c_{1 i}^{2}}{2 \Upsilon_{P_{i}}^{2}}-\Upsilon_{P_{i}} & =0
\end{aligned}
$$

Then

$$
\begin{aligned}
c_{1 i} & =v_{i} \Upsilon_{P_{i}} \\
\frac{\left(v_{i} \Upsilon_{P_{i}}\right)^{2}}{2 \Upsilon_{P_{i}}^{2}}-\Upsilon_{P_{i}} & =0 \\
\Upsilon_{P_{i}} & =\frac{\left(v_{i}\right)^{2}}{2} \\
c_{1 i} & =\frac{\left(v_{i}\right)^{3}}{2}
\end{aligned}
$$

The variation explored in this section delivers an interesting new insight: when the quality of parental involvement is endogenous, the strength of rewards becomes more sensitive to student talent.

\section{A.5 Other population targets}

Headmasters care about the learning outcomes of a whole distribution of students attending their school. In our main analysis, we assumed that the relevant moment for the headmasters was the mean of the outcome distribution. This is, of course, a crude description of the reality and headmasters (as well as policy makers) target different measures of educational achievement. A more general expression could be obtained by assuming that the headmaster cares about a weighted average of the average student and the $n$-th order statistic of the human capital distribution $\left(H_{i}\right)$ at school $j$, denoted $H_{j}^{(n)}$. Then, let $v_{j}^{(n)}$ be the talent corresponding to $H_{j}^{(n)}$ and assume

$$
U_{H M j}=\lambda \frac{1}{N_{j}} \sum_{i \in j} H_{i}+(1-\lambda) H_{j}^{(n)}+\left(T \gamma-\frac{n_{j}}{N_{j}} \sum_{i \in j} \frac{c_{2 j}\left(c_{1 i}+c_{2 j}\right)}{2 \Upsilon_{T_{j}}}\right) .
$$

The parameter $\lambda$, with $\lambda \in[0,1]$, characterizes the weight given to each goal. Following the analysis at the core of the paper we obtain that the equilibrium individual effort is given by: 
$e_{i}=\Upsilon_{P_{i}} v_{i}+\frac{1}{3} \Upsilon_{P_{i_{M}}} v_{i_{M}}\left(\frac{\left(\frac{2 \Upsilon_{T_{j}}\left(\lambda \bar{v}_{j}+(1-\lambda) v_{j}^{(n)}\right)}{\Upsilon_{T_{j M}}\left(\lambda \bar{v}_{j_{M}}+(1-\lambda) v_{j_{M}}^{(n)}\right)}+\theta_{j}\right)\left(\frac{2 \Upsilon_{T_{j M}}\left(\lambda \bar{v}_{j_{M}}+(1-\lambda) v_{j_{M}}^{(n)}\right)}{3}\right)^{2} \frac{1}{\Upsilon_{P_{i M}}}-\theta_{j} \omega}{\left(\omega-\left(\frac{2 \Upsilon_{T_{j M}}\left(\lambda \bar{v}_{j_{M}}+(1-\lambda) v_{j_{M}}^{(n)}\right)}{3}\right)^{2} \frac{1}{\Upsilon_{P_{i M}}}\right)}\right)$

This expression is similar to 22 since it relates individual student effort to the achievement of the targeted goal at the school level and at the school attended by the median child. Importantly, this means that any measure of inequality that is captured by an order statistic may be easily accommodated within our formulation, and the nature of the endogenous peer-effects are qualitatively unchanged, although they would depend on different features of the classroom composition. Notice, however, that other measures of human capital inequality based on different moments of the distribution would be more difficult to handle analytically, although this result suggests that it is unlikely they would yield qualitatively different implications.

\section{A.6 Relative performance evaluation as the target}

What would happen to school motivation effort if the headmaster has the goal of achieving a certain average student outcome? Let $H_{R}$ be the human capital of average student in the reference $(R)$ school and assume that:

$$
U_{H M j}=\kappa I_{\frac{1}{N_{j}} \sum_{i \in j}\left(c_{1 i}+c_{2 j}\right) v_{i} \geq H_{R}}+\left(T \gamma-\frac{n_{j}}{N_{j}} \sum_{i \in j} \frac{c_{2 j}\left(c_{1 i}+c_{2 j}\right)}{2 \Upsilon_{T_{j}}}\right)
$$

under these circumstances in equilibrium the effort of the $c_{2 j}$ is

$$
c_{2 j}=\frac{H_{R}-\frac{1}{N_{j}} \sum_{i \in j} c_{1 i} v_{i}}{\bar{v}_{j}}
$$

iff

$$
\kappa \geq \frac{n_{j}}{N_{j}} \sum_{i \in j} \frac{\frac{H_{R}-\frac{1}{N_{j}} \sum_{i \in j} c_{1 i} v_{i}}{\bar{v}_{j}}\left(c_{1 i}+\frac{H_{R}-\frac{1}{N_{j}} \sum_{i \in j} c_{1 i} v_{i}}{\bar{v}_{j}}\right)}{2 \Upsilon_{T_{j}}}
$$

Since in equilibrium

$$
c_{1 i} v_{i}=\Upsilon_{P_{i}} v_{i}^{2}-\frac{H_{R}-\frac{1}{N_{j}} \sum_{k \in j} c_{1 k} v_{k}}{2 \bar{v}_{j}} v_{i}
$$


we have (after some algebra) that

$$
\frac{1}{N_{j}} \sum_{k \in j} c_{1 k} v_{k}=\frac{2}{3 N_{j}} \sum_{k \in j} \Upsilon_{P_{k}} v_{k}^{2}-\frac{1}{3} H_{R}
$$

so $c_{2 j}$ is positive iff

$$
\kappa \geq \frac{n_{j}}{N_{j}} \sum_{i \in j} \frac{\frac{\frac{2}{3} H_{R}-\frac{2}{3 N_{j}} \sum_{k \in j} \Upsilon_{P_{k}} v_{k}^{2}}{\bar{v}_{j}}\left(c_{1 i}+\frac{\frac{2}{3} H_{R}-\frac{2}{3 N_{j}} \sum_{k \in j} \Upsilon_{P_{k}} v_{k}^{2}}{\bar{v}_{j}}\right)}{2 \Upsilon_{T_{j}}}
$$

Notice that the effect of increasing the target is to increase effort for those schools which are in a position to achieve it, but it decreases the effort to zero for those with low types.

\section{B Appendix: Proofs}

\section{Proof of lemma 1}

Proof Notice that the preferences of an arbitrary parent $i$ with respect to a class size level $n$ (once he takes into account the taxes that the policymaker will have to levy to pay for the costs of such class size) is:

$$
\begin{aligned}
U_{P_{i}}= & \left(\Upsilon_{P_{i}} v_{i}+\frac{1}{3}\left(\frac{2}{n} \Upsilon_{T_{j M}} \bar{v}_{j}-\bar{\Omega}_{j}\right)\right) v_{i}+ \\
& +\left(T \psi_{i}-\frac{1}{2 \Upsilon_{P_{i}}}\left(\left(\Upsilon_{P_{i}} v_{i}\right)^{2}-\frac{1}{9}\left(\frac{2}{n} \Upsilon_{T_{j}} \bar{v}_{j}-\bar{\Omega}_{j}\right)^{2}\right)\right)-\frac{\omega}{2 n^{2}},
\end{aligned}
$$

so that

$$
\begin{aligned}
\operatorname{sign}\left(\frac{\partial U_{P_{i}}}{\partial n}\right) & =\operatorname{sign}\left(-\frac{v_{i}}{3}\left(\frac{2 \Upsilon_{T_{j}} \bar{v}_{j}}{n^{2}}\right)-\frac{2 \Upsilon_{T_{j}} \bar{v}_{j}}{9 \Upsilon_{P_{i}} n^{2}}\left(\frac{2}{n} \Upsilon_{T_{j}} \bar{v}_{j}-\bar{\Omega}_{j}\right)+\frac{\omega}{n^{3}}\right) \\
& =\operatorname{sign}\left(\left(-\frac{2 v_{i} \Upsilon_{T_{j}} \bar{v}_{j}}{3}+\bar{\Omega}_{j} \frac{2 \Upsilon_{T_{j}} \bar{v}_{j}}{9 \Upsilon_{P_{i}}}\right) n-\frac{4 \Upsilon_{T_{j}}^{2} \bar{v}_{j}^{2}}{9 \Upsilon_{P_{i}}}+\omega\right)
\end{aligned}
$$

and therefore the sign of the derivative of $U_{P_{i}}$ with respect to $n$ can change sign only once. This means that there is at most one interior critical point. For this critical point to be a maximum it is sufficient that 


$$
\begin{aligned}
-\frac{2 v_{i} \Upsilon_{T_{j}} \bar{v}_{j}}{3}+\bar{\Omega}_{j} \frac{2 \Upsilon_{T_{j}} \bar{v}_{j}}{9 \Upsilon_{P_{i}}} & <0 \\
\bar{\Omega}_{j} & =\frac{1}{N_{j}} \sum_{i \in j} \Upsilon_{P_{i}} v_{i}<3 \Upsilon_{P_{i}} v_{i}
\end{aligned}
$$

And this is verified if

$$
\frac{1}{N_{j}} \sum_{i \in j} \Upsilon_{P_{i}} v_{i}<3 \min _{i \in\{1, \ldots, N\}} \Upsilon_{P_{i}} v_{i}
$$




\section{Online Appendix C: Algebra (not intended for publica- tion)}

$n$ derivation

$$
U_{P M}=\frac{2 v^{2}}{3}\left(\Upsilon_{P}+\frac{\Upsilon_{T}}{n}\right)+\left(T \psi-\frac{2 v^{2}}{9 \Upsilon_{P}}\left(2 \Upsilon_{P}^{2}+\frac{\Upsilon_{P} \Upsilon_{T}}{n}-\frac{\Upsilon_{T}^{2}}{n^{2}}\right)\right)-\frac{\omega}{2} \frac{1}{n^{2}}
$$

The first order condition with respect to $n$ is:

$$
\begin{aligned}
\frac{\partial U_{P M}}{\partial n}=- & \frac{2 v^{2}}{3}\left(\frac{\Upsilon_{T}}{n^{2}}\right)-\frac{2 v^{2}}{9 \Upsilon_{P}}\left(-\frac{\Upsilon_{P} \Upsilon_{T}}{n^{2}}+2 \frac{\Upsilon_{T}^{2}}{n^{3}}\right)+\omega \frac{1}{n^{3}} \\
n^{2} \frac{\partial U_{P M}}{\partial n} & =-\frac{2 v^{2}}{3} \Upsilon_{T}-\frac{2 v^{2}}{9 \Upsilon_{P}}\left(-\Upsilon_{P} \Upsilon_{T}+2 \frac{\Upsilon_{T}^{2}}{n}\right)+\omega \frac{1}{n} \\
& =-\frac{2 v^{2}}{3} \Upsilon_{T}+\frac{2 v^{2}}{9} \Upsilon_{T}-\frac{4 v^{2}}{9 \Upsilon_{P}} \frac{\Upsilon_{T}^{2}}{n}+\omega \frac{1}{n} \\
& =-\frac{4 v^{2}}{9} \Upsilon_{T}-\frac{4 v^{2}}{9 \Upsilon_{P}} \frac{\Upsilon_{T}^{2}}{n}+\omega \frac{1}{n}
\end{aligned}
$$

Thus the only critical point is:

$$
n=\frac{\omega-\frac{4 v^{2}}{9 \Upsilon_{P}} \Upsilon_{T}^{2}}{\frac{4 v^{2}}{9} \Upsilon_{T}}
$$

\section{Comparative statics (homogeneous case)}

Comparative statics for $c_{1}, c_{2}$, and $e$ with respect to $v$

$$
\begin{aligned}
c_{1} & =\frac{2 v \Upsilon_{P}}{3}\left(\frac{2 \omega-3\left(\frac{2 v \Upsilon_{T}}{3}\right)^{2} \frac{1}{\Upsilon_{P}}}{\omega-\left(\frac{2 v \Upsilon_{T}}{3}\right)^{2} \frac{1}{\Upsilon_{P}}}\right) \\
& =\frac{2 \Upsilon_{P}}{3}\left(\frac{2 \omega v-3\left(\frac{2 \Upsilon_{T}}{3}\right)^{2} \frac{v^{3}}{\Upsilon_{P}}}{\omega-\left(\frac{2 v \Upsilon_{T}}{3}\right)^{2} \frac{1}{\Upsilon_{P}}}\right)
\end{aligned}
$$




$$
\begin{aligned}
\frac{\partial c_{1}}{\partial v} & =\frac{2 \Upsilon_{P}}{3} \frac{\left[2 \omega-9\left(\frac{2 v \Upsilon_{T}}{3}\right)^{2} \frac{1}{\Upsilon_{P}}\right]\left[\omega-\left(\frac{2 v \Upsilon_{T}}{3}\right)^{2} \frac{1}{\Upsilon_{P}}\right]+\left[2 \omega v-3\left(\frac{2 v \Upsilon_{T}}{3}\right)^{2} \frac{1}{\Upsilon_{P}} v\right]\left(\frac{2 \Upsilon_{T}}{3}\right)^{2} 2 v \frac{1}{\Upsilon_{P}}}{\left[\omega-\left(\frac{2 v \Upsilon_{T}}{3}\right)^{2} \frac{1}{\Upsilon_{P}}\right]^{2}} \\
& =\frac{2 \Upsilon_{P}}{3} \frac{\left[2 \omega-9\left(\frac{2 v \Upsilon_{T}}{3}\right)^{2} \frac{1}{\Upsilon_{P}}\right]\left[\omega-\left(\frac{2 v \Upsilon_{T}}{3}\right)^{2} \frac{1}{\Upsilon_{P}}\right]+\left[4 \omega-6\left(\frac{2 v \Upsilon_{T}}{3}\right)^{2} \frac{1}{\Upsilon_{P}}\right]\left(\frac{2 v \Upsilon_{T}}{3}\right)^{2} \frac{1}{\Upsilon_{P}}}{\left[\omega-\left(\frac{2 v \Upsilon_{T}}{3}\right)^{2} \frac{1}{\Upsilon_{P}}\right]^{2}} \\
& =\frac{2 \Upsilon_{P}}{3} \frac{\left(\frac{2 v \Upsilon_{T}}{3}\right)^{2} \frac{1}{\Upsilon_{P}}\left[\left(4 \omega-6\left(\frac{2 v \Upsilon_{T}}{3}\right)^{2} \frac{1}{\Upsilon_{P}}\right)-\left(2 \omega-9\left(\frac{2 v \Upsilon_{T}}{3}\right)^{2} \frac{1}{\Upsilon_{P}}\right)\right]+\omega\left[2 \omega-9\left(\frac{2 v \Upsilon_{T}}{3}\right)^{2} \frac{1}{\Upsilon_{P}}\right]}{\left[\omega-\left(\frac{2 v \Upsilon_{T}}{3}\right)^{2} \frac{1}{\Upsilon_{P}}\right]^{2}} \\
& =\frac{2 \Upsilon_{P}}{3} \frac{\left(\frac{2 v \Upsilon_{T}}{3}\right)^{2} \frac{1}{\Upsilon_{P}}\left[2 \omega+3\left(\frac{2 v \Upsilon_{T}}{3}\right)^{2} \frac{1}{\Upsilon_{P}}\right]+\omega\left[2 \omega-9\left(\frac{2 v \Upsilon_{T}}{3}\right)^{2} \frac{1}{\Upsilon_{P}}\right]}{\left[\omega-\left(\frac{2 v \Upsilon_{T}}{3}\right)^{2} \frac{1}{\Upsilon_{P}}\right]^{2}} \\
& =\frac{2 \Upsilon_{P}}{3} \frac{3\left(\frac{2 v \Upsilon_{T}}{3}\right)^{4} \frac{1}{\Upsilon_{P}^{2}}+\omega\left[2 \omega-7\left(\frac{2 v \Upsilon_{T}}{3}\right)^{2} \frac{1}{\Upsilon_{P}}\right]}{\left[\omega-\left(\frac{2 v \Upsilon_{T}}{3}\right)^{2} \frac{1}{\Upsilon_{P}}\right]^{2}}
\end{aligned}
$$

and $c_{2}>0$ implies that:

$$
2 \omega<6\left(\frac{2 v \Upsilon_{T}}{3}\right)^{2} \frac{1}{\Upsilon_{P}}
$$

and hence

$$
2 \omega<6\left(\frac{2 v \Upsilon_{T}}{3}\right)^{2} \frac{1}{\Upsilon_{P}}+\left(\frac{2 v \Upsilon_{T}}{3}\right)^{2} \frac{1}{\Upsilon_{P}}
$$

which leaves $\frac{\partial c_{1}}{\partial v}>0$.

$$
\begin{aligned}
c_{2} & =\frac{2 v \Upsilon_{P}}{3}\left(\frac{3\left(\frac{2 v \Upsilon_{T}}{3}\right)^{2} \frac{1}{\Upsilon_{P}}-\omega}{\omega-\left(\frac{2 v \Upsilon_{T}}{3}\right)^{2} \frac{1}{\Upsilon_{P}}}\right) \\
& =\frac{2 \Upsilon_{P}}{3}\left(\frac{3\left(\frac{2 \Upsilon_{T}}{3}\right)^{2} \frac{1}{\Upsilon_{P}} v^{3}-\omega v}{\omega-\left(\frac{2 v \Upsilon_{T}}{3}\right)^{2} \frac{1}{\Upsilon_{P}}}\right)
\end{aligned}
$$




$$
\begin{aligned}
\frac{\partial c_{2}}{\partial v} & =\frac{2 \Upsilon_{P}}{3} \frac{\left[9\left(\frac{2 \Upsilon_{T}}{3}\right)^{2} \frac{1}{\Upsilon_{P}} v^{2}-\omega\right]\left[\omega-\left(\frac{2 v \Upsilon_{T}}{3}\right)^{2} \frac{1}{\Upsilon_{P}}\right]+\left[3\left(\frac{2 \Upsilon_{T}}{3}\right)^{2} \frac{1}{\Upsilon_{P}} v^{3}-\omega v\right]\left(\frac{2 \Upsilon_{T}}{3}\right)^{2} 2 v \frac{1}{\Upsilon_{P}}}{\left[\omega-\left(\frac{2 v \Upsilon_{T}}{3}\right)^{2} \frac{1}{\Upsilon_{P}}\right]^{2}} \\
& =\frac{2 \Upsilon_{P}}{3} \frac{\left[9\left(\frac{2 v \Upsilon_{T}}{3}\right)^{2} \frac{1}{\Upsilon_{P}}-\omega\right]\left[\omega-\left(\frac{2 v \Upsilon_{T}}{3}\right)^{2} \frac{1}{\Upsilon_{P}}\right]+\left[3\left(\frac{2 v \Upsilon_{T}}{3}\right)^{2} \frac{1}{\Upsilon_{P}}-\omega\right]\left(\frac{2 v \Upsilon_{T}}{3}\right)^{2} 2 \frac{1}{\Upsilon_{P}}}{\left[\omega-\left(\frac{2 v \Upsilon_{T}}{3}\right)^{2} \frac{1}{\Upsilon_{P}}\right]^{2}}
\end{aligned}
$$

but $c_{1}>0$ implies

$$
\omega>\left(\frac{2 v \Upsilon_{T}}{3}\right)^{2} \frac{1}{\Upsilon_{P}}
$$

and $c_{2}>0$ implies

$$
3\left(\frac{2 v \Upsilon_{T}}{3}\right)^{2} \frac{1}{\Upsilon_{P}}>\omega
$$

and therefore that

$$
9\left(\frac{2 v \Upsilon_{T}}{3}\right)^{2} \frac{1}{\Upsilon_{P}}>\omega
$$

Then, $\frac{\partial c_{2}}{\partial v}>0$.

$$
\begin{gathered}
e=\frac{\frac{2 v \omega \Upsilon_{P}}{3}}{\omega-\left(\frac{2 v \Upsilon_{T}}{3}\right)^{2} \frac{1}{\Upsilon_{P}}} \\
\frac{\partial e}{\partial v}=\frac{\frac{2 \omega \Upsilon_{P}}{3}\left[\omega-\left(\frac{2 v \Upsilon_{T}}{3}\right)^{2} \frac{1}{\Upsilon_{P}}\right]+2\left(\frac{2 v \Upsilon_{T}}{3}\right)^{2} \frac{1}{\Upsilon_{P}} \frac{2 \omega \Upsilon_{P}}{3}}{\left[\omega-\left(\frac{2 v \Upsilon_{T}}{3}\right)^{2} \frac{1}{\Upsilon_{P}}\right]^{2}} \\
=\frac{\frac{2 \omega \Upsilon_{P}}{3}\left[\omega+\left(\frac{2 v \Upsilon_{T}}{3}\right)^{2} \frac{1}{\Upsilon_{P}}\right]}{\left[\omega-\left(\frac{2 v \Upsilon_{T}}{3}\right)^{2} \frac{1}{\Upsilon_{P}}\right]^{2}}>0 .
\end{gathered}
$$

Comparative statics for $c_{1}, c_{2}$, and $e$ with respect to $\varpi$

$$
c_{1}=\frac{2 v \Upsilon_{P}}{3}\left(\frac{2 \omega-3\left(\frac{2 v \Upsilon_{T}}{3}\right)^{2} \frac{1}{\Upsilon_{P}}}{\omega-\left(\frac{2 v \Upsilon_{T}}{3}\right)^{2} \frac{1}{\Upsilon_{P}}}\right)
$$




$$
\begin{aligned}
& \frac{\partial c_{1}}{\partial \omega}=\frac{2 v \Upsilon_{P}}{3} \frac{2\left[\omega-\left(\frac{2 v \Upsilon_{T}}{3}\right)^{2} \frac{1}{\Upsilon_{P}}\right]-\left[2 \omega-3\left(\frac{2 v \Upsilon_{T}}{3}\right)^{2} \frac{1}{\Upsilon_{P}}\right]}{\left[\omega-\left(\frac{2 v \Upsilon_{T}}{3}\right)^{2} \frac{1}{\Upsilon_{P}}\right]^{2}} \\
& =\frac{2 v \Upsilon_{P}}{3} \frac{\left(\frac{2 v \Upsilon_{T}}{3}\right)^{2} \frac{1}{\Upsilon_{P}}}{\left[\omega-\left(\frac{2 v \Upsilon_{T}}{3}\right)^{2} \frac{1}{\Upsilon_{P}}\right]^{2}}>0 \\
& c_{2}=\frac{2 v \Upsilon_{P}}{3}\left(\frac{3\left(\frac{2 v \Upsilon_{T}}{3}\right)^{2} \frac{1}{\Upsilon_{P}}-\omega}{\omega-\left(\frac{2 v \Upsilon_{T}}{3}\right)^{2} \frac{1}{\Upsilon_{P}}}\right) \\
& \frac{\partial c_{2}}{\partial \omega}=\frac{2 v \Upsilon_{P}}{3} \frac{-\left[\omega-\left(\frac{2 v \Upsilon_{T}}{3}\right)^{2} \frac{1}{\Upsilon_{P}}\right]-\left[3\left(\frac{2 v \Upsilon_{T}}{3}\right)^{2} \frac{1}{\Upsilon_{P}}-\omega\right]}{\left[\omega-\left(\frac{2 v \Upsilon_{T}}{3}\right)^{2} \frac{1}{\Upsilon_{P}}\right]^{2}} \\
& =-\frac{2 v \Upsilon_{P}}{3} \frac{2\left(\frac{2 v \Upsilon_{T}}{3}\right)^{2} \frac{1}{\Upsilon_{P}}}{\left[\omega-\left(\frac{2 v \Upsilon_{T}}{3}\right)^{2} \frac{1}{\Upsilon_{P}}\right]^{2}}<0 \\
& e=\frac{\frac{2 v \omega \Upsilon_{P}}{3}}{\omega-\left(\frac{2 v \Upsilon_{T}}{3}\right)^{2} \frac{1}{\Upsilon_{P}}} \\
& \frac{\partial e}{\partial \omega}=\frac{\frac{2 v \Upsilon_{P}}{3}\left[\omega-\left(\frac{2 v \Upsilon_{T}}{3}\right)^{2} \frac{1}{\Upsilon_{P}}\right]-\frac{2 v \omega \Upsilon_{P}}{3}}{\left[\omega-\left(\frac{2 v \Upsilon_{T}}{3}\right)^{2} \frac{1}{\Upsilon_{P}}\right]^{2}} \\
& =\frac{-\left(\frac{2 v \Upsilon_{T}}{3}\right)^{2} \frac{1}{\Upsilon_{P}}}{\left[\omega-\left(\frac{2 v \Upsilon_{T}}{3}\right)^{2} \frac{1}{\Upsilon_{P}}\right]^{2}}<0
\end{aligned}
$$

Comparative statics for $c_{1}, c_{2}$, and $e$ with respect to $\Upsilon_{P}$

$$
\begin{aligned}
c_{1} & =\frac{2 v \Upsilon_{P}}{3}\left(\frac{2 \omega-3\left(\frac{2 v \Upsilon_{T}}{3}\right)^{2} \frac{1}{\Upsilon_{P}}}{\omega-\left(\frac{2 v \Upsilon_{T}}{3}\right)^{2} \frac{1}{\Upsilon_{P}}}\right) \\
& =\frac{2 v}{3}\left(\frac{2 \omega \Upsilon_{P}^{2}-3\left(\frac{2 v \Upsilon_{T}}{3}\right)^{2} \Upsilon_{P}}{\omega \Upsilon_{P}^{2}-\left(\frac{2 v \Upsilon_{T}}{3}\right)^{2} \Upsilon_{P}}\right)
\end{aligned}
$$




$$
\begin{gathered}
\frac{\partial c_{1}}{\partial \Upsilon_{P}}=\frac{2 v}{3} \frac{\left(4 \omega \Upsilon_{P}-3\left(\frac{2 v \Upsilon_{T}}{3}\right)^{2}\right)\left(\omega \Upsilon_{P}^{2}-\left(\frac{2 v \Upsilon_{T}}{3}\right)^{2} \Upsilon_{P}\right)-\left(2 \omega \Upsilon_{P}-\left(\frac{2 v \Upsilon_{T}}{3}\right)^{2}\right)\left(2 \omega \Upsilon_{P}^{2}-3\left(\frac{2 v \Upsilon_{T}}{3}\right)^{2} \Upsilon_{P}\right)}{\left(\omega \Upsilon_{P}^{2}-\left(\frac{2 v \Upsilon_{T}}{3}\right)^{2} \Upsilon_{P}\right)^{2}} \\
=\frac{2 v}{3} \frac{4 \omega^{2} \Upsilon_{P}^{3}-\frac{28}{9} \omega \Upsilon_{P}^{2} v^{2} \Upsilon_{T}^{2}+\frac{16}{27} \Upsilon_{P} v^{4} \Upsilon_{T}^{4}-\left(4 \omega^{2} \Upsilon_{P}^{3}-\frac{32}{9} \omega \Upsilon_{P}^{2} v^{2} \Upsilon_{T}^{2}+\frac{16}{27} \Upsilon_{P} v^{4} \Upsilon_{T}^{4}\right)}{\left(\omega \Upsilon_{P}^{2}-\left(\frac{2 v \Upsilon_{T}}{3}\right)^{2} \Upsilon_{P}\right)^{2}} \\
=\frac{2 v}{3} \frac{\frac{4}{9} \omega \Upsilon_{P}^{2} v^{2} \Upsilon_{T}^{2}}{\left(\omega \Upsilon_{P}^{2}-\left(\frac{2 v \Upsilon_{T}}{3}\right)^{2} \Upsilon_{P}\right)^{2}}=\frac{2 v}{3} \frac{\frac{4}{9} \omega v^{2} \Upsilon_{T}^{2}}{\left(\omega \Upsilon_{P}-\left(\frac{2 v \Upsilon_{T}}{3}\right)^{2}\right)^{2}}>0 \\
c_{2}=\frac{2 v \Upsilon_{P}}{3}\left(\frac{3\left(\frac{2 v \Upsilon_{T}}{3}\right)^{2} \frac{1}{\Upsilon_{P}}-\omega}{\omega-\left(\frac{2 v \Upsilon_{T}}{3}\right)^{2} \frac{1}{\Upsilon_{P}}}\right) \\
=\frac{2 v}{3}\left(\frac{3\left(\frac{2 v \Upsilon_{T}}{3}\right)^{2}-\omega \Upsilon_{P}}{\omega \Upsilon_{P}-\left(\frac{2 v \Upsilon_{T}}{3}\right)^{2}}\right)
\end{gathered}
$$

Notice the numerator is decreasing in $\Upsilon_{P}$ and the denominator increasing in $\Upsilon_{P}$, thus

$$
\begin{gathered}
\frac{\partial c_{2}}{\partial \Upsilon_{P}}<0 \\
e=\frac{\frac{2 v \omega \Upsilon_{P}}{3}}{\omega-\left(\frac{2 v \Upsilon_{T}}{3}\right)^{2} \frac{1}{\Upsilon_{P}}}=\frac{2 v \omega \Upsilon_{P}^{2}}{3 \omega \Upsilon_{P}-3\left(\frac{2 v \Upsilon_{T}}{3}\right)^{2}} \\
\frac{\partial e}{\partial \Upsilon_{P}}=\frac{4 v \omega \Upsilon_{P}\left(3 \omega \Upsilon_{P}-3\left(\frac{2 v \Upsilon_{T}}{3}\right)^{2}\right)-6 v \omega^{2} \Upsilon_{P}^{2}}{\left(3 \omega \Upsilon_{P}-3\left(\frac{2 v \Upsilon_{T}}{3}\right)^{2}\right)^{2}}=\frac{\frac{2}{3} v \omega \Upsilon_{P}\left(9 \omega \Upsilon_{P}-8 v^{2} \Upsilon_{T}^{2}\right)}{\left(3 \omega \Upsilon_{P}-3\left(\frac{2 v \Upsilon_{T}}{3}\right)^{2}\right)^{2}} \lesseqgtr 0
\end{gathered}
$$

The effect is negative if $\omega<\frac{8 v^{2} \Upsilon_{T}^{2}}{9 \Upsilon_{P}}$.

Comparative statics for $c_{1}, c_{2}$, and $e$ with respect to $\Upsilon_{T}$

$$
\begin{aligned}
c_{1} & =\frac{2 v \Upsilon_{P}}{3}\left(\frac{2 \omega-3\left(\frac{2 v \Upsilon_{T}}{3}\right)^{2} \frac{1}{\Upsilon_{P}}}{\omega-\left(\frac{2 v \Upsilon_{T}}{3}\right)^{2} \frac{1}{\Upsilon_{P}}}\right) \\
& =\frac{2 v}{3}\left(\frac{2 \omega \Upsilon_{P}^{2}-3\left(\frac{2 v \Upsilon_{T}}{3}\right)^{2} \Upsilon_{P}}{\omega \Upsilon_{P}^{2}-\left(\frac{2 v \Upsilon_{T}}{3}\right)^{2} \Upsilon_{P}}\right)
\end{aligned}
$$




$$
\begin{gathered}
\frac{\partial c_{1}}{\partial \Upsilon_{T}}=\frac{2 v}{3} \frac{-\frac{8 v^{2} \Upsilon_{T}}{3} \Upsilon_{P}\left(\omega \Upsilon_{P}^{2}-\left(\frac{2 v \Upsilon_{T}}{3}\right)^{2} \Upsilon_{P}\right)+\frac{8 v^{2} \Upsilon_{T}}{9} \Upsilon_{P}\left(2 \omega \Upsilon_{P}^{2}-3\left(\frac{2 v \Upsilon_{T}}{3}\right)^{2} \Upsilon_{P}\right)}{\left(\omega \Upsilon_{P}^{2}-\left(\frac{2 v \Upsilon_{T}}{3}\right)^{2} \Upsilon_{P}\right)^{2}} \\
=\frac{2 v}{3} \frac{-\frac{8}{9} \omega v^{2} \Upsilon_{P}^{3} \Upsilon_{T}}{\left(\omega \Upsilon_{P}^{2}-\left(\frac{2 \Upsilon_{T}}{3}\right)^{2} \Upsilon_{P}\right)^{2}}<0 \\
c_{2}=\frac{2 v \Upsilon_{P}}{3}\left(\frac{3\left(\frac{2 v \Upsilon_{T}}{3}\right)^{2} \frac{1}{\Upsilon_{P}}-\omega}{\omega-\left(\frac{2 v \Upsilon_{T}}{3}\right)^{2} \frac{1}{\Upsilon_{P}}}\right) \\
=\frac{2 v}{3}\left(\frac{3\left(\frac{2 v \Upsilon_{T}}{3}\right)^{2}-\omega \Upsilon_{P}}{\omega \Upsilon_{P}-\left(\frac{2 v \Upsilon_{T}}{3}\right)^{2}}\right)
\end{gathered}
$$

Notice the numerator is increasing in $\Upsilon_{T}$ and the denominator decreasing in $\Upsilon_{T}$, thus

$$
\begin{gathered}
\frac{\partial c_{2}}{\partial \Upsilon_{T}}>0 \\
e=\frac{\frac{2 v \omega \Upsilon_{P}}{3}}{\omega-\left(\frac{2 v \Upsilon_{T}}{3}\right)^{2} \frac{1}{\Upsilon_{P}}}=\frac{2 v \omega \Upsilon_{P}^{2}}{3 \omega \Upsilon_{P}-3\left(\frac{2 v \Upsilon_{T}}{3}\right)^{2}}
\end{gathered}
$$

Notice the the denominator decreasing in $\Upsilon_{T}$, thus

$$
\frac{\partial e}{\partial \Upsilon_{T}}<0
$$

